\title{
Stereospecific $\mathrm{Pd}(\mathrm{O})$-Catalyzed Malonate Additions
}

\author{
to Allylic Hydroxy Phosphonate Derivatives: A \\ Formal Synthesis of (-) Enterolactone
}

\author{
Bingli Yan and Christopher D. Spilling* \\ Department of Chemistry and Biochemistry, University of Missouri-St. Louis, \\ 8001 Natural Bridge Road, St. Louis, Missouri 63121. \\ cspill@umsl.edu
}

\section{Supporting Material}

Table of Contents:

$\begin{array}{ll}\text { General experimental } & \text { S2 }\end{array}$

$\begin{array}{ll}\text { Experimental procedure and characterization data for }( \pm) \mathbf{1 a} & \mathrm{S} 3\end{array}$

$\begin{array}{ll}\text { Experimental procedure for }(\boldsymbol{R}) \mathbf{1 a} & \text { S3 }\end{array}$

$\begin{array}{ll}\text { Experimental procedure and characterization data for } \mathbf{3 a} & \text { S3 }\end{array}$

$\begin{array}{ll}\text { Experimental procedure and characterization data for }(\boldsymbol{R}) \mathbf{1 b} & \mathrm{S} 4\end{array}$

Experimental procedure and characterization data for $\mathbf{3 b} \quad$ S5

Experimental procedure and characterization data for $6 \quad$ S5

Experimental procedure for the formation of $(1 R)(2 E)$ Dimethyl [1-(methoxycarbonyloxy)-4-

(3-methoxyphenyl)-2-butenyl]phosphonate 3d from Phosphonate 1b S6

A General Procedure for the Addition of Malonates to Allylic Carbonates 3b-d $\quad$ S7

$\begin{array}{ll}\text { Experimental procedure and characterization data for 11a } & \text { S7 }\end{array}$ 
$\begin{array}{ll}\text { Experimental procedure and characterization data for } \mathbf{1 1 b} & \mathrm{S} 8\end{array}$

$\begin{array}{ll}\text { Experimental procedure and characterization data for } \mathbf{1 2} & \text { S8 }\end{array}$

$\begin{array}{ll}\text { Experimental procedure and characterization data for 11c } & \text { S9 }\end{array}$

References $\quad$ S9

$\begin{array}{lr}{ }^{1} \mathrm{H} \text { and }{ }^{13} \mathrm{C} \text { NMR spectra for 1a } & \mathrm{S} 10\end{array}$

${ }^{1} \mathrm{H}$ and ${ }^{13} \mathrm{C}$ NMR spectra for 3a $\quad$ S11

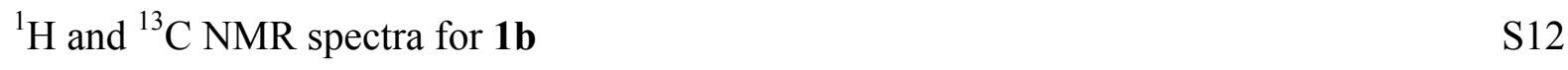

${ }^{1} \mathrm{H}$ and ${ }^{13} \mathrm{C}$ NMR spectra for $\mathbf{3 b} \quad \mathrm{S} 13$

$\begin{array}{lr}{ }^{1} \mathrm{H} \text { and }{ }^{13} \mathrm{C} \text { NMR spectra for } \mathbf{6} & \mathrm{S} 14\end{array}$

$\begin{array}{ll}{ }^{1} \mathrm{H} \text { and }{ }^{13} \mathrm{C} \text { NMR spectra for } \mathbf{3 d} & \mathrm{S} 15\end{array}$

$\begin{array}{lr}{ }^{1} \mathrm{H} \text { and }{ }^{13} \mathrm{C} \text { NMR spectra for 11a } & \mathrm{S} 16\end{array}$

$\begin{array}{lr}{ }^{1} \mathrm{H} \text { and }{ }^{13} \mathrm{C} \text { NMR spectra for } \mathbf{1 1 b} & \mathrm{S} 17\end{array}$

$\begin{array}{lr}{ }^{1} \mathrm{H} \text { and }{ }^{13} \mathrm{C} \text { NMR spectra for } \mathbf{1 2} & \mathrm{S} 18\end{array}$

$\begin{array}{lr}{ }^{1} \mathrm{H} \text { and }{ }^{13} \mathrm{C} \text { NMR spectra for 11e } & \text { S19 }\end{array}$

$\begin{array}{lr}{ }^{1} \mathrm{H} \text { and }{ }^{13} \mathrm{C} \text { NMR spectra for 11d } & \text { S20 }\end{array}$

$\begin{array}{ll}{ }^{1} \mathrm{H} \text { and }{ }^{13} \mathrm{C} \text { NMR spectra for } \mathbf{1 3} & \text { S21 }\end{array}$

${ }^{1} \mathrm{H}$ and ${ }^{13} \mathrm{C}$ NMR spectra for $\mathbf{1 4} \quad$ S22

$\begin{array}{lr}\text { HPLC data for }( \pm) \text { and non racemic } \mathbf{1 b} & \text { S24 }\end{array}$

$\begin{array}{lr}\text { HPLC data for }( \pm) \text { and non racemic } \mathbf{3 b} & \text { S24 }\end{array}$

HPLC data for $( \pm)$ and non racemic 11a $\quad$ S25

$\begin{array}{lr}\text { HPLC data for }( \pm) \text { and non racemic 3d } & \text { S26 }\end{array}$

$\begin{array}{lr}\text { HPLC data for }( \pm) \text { and non racemic } \mathbf{1 4} & \text { S27 }\end{array}$

General Experimental: ${ }^{1} \mathrm{H},{ }^{13} \mathrm{C}$, and ${ }^{31} \mathrm{P}$ NMR spectra were recorded at 300,75 and $121 \mathrm{MHz}$, repectively in $\mathrm{CDCl}_{3} .{ }^{1} \mathrm{H}$ NMR spectra are referenced to internal tetramethylsilane (TMS, $\delta=$ 0.00) or $\mathrm{CHCl}_{3}(7.27 \mathrm{ppm}),{ }^{13} \mathrm{C}$ NMR spectra to the center-line of $\mathrm{CDCl}_{3}(77.23 \mathrm{ppm})$ and ${ }^{31} \mathrm{P}$ NMR spectra to external $85 \% \mathrm{H}_{3} \mathrm{PO}_{4}$. Coupling constants, $J$, are reported in Hz. Enantiomer ratios were measured by chiral stationary-phase HPLC on a $(S, S)$-Whelk-O column, a ChiralPak 
AS or AD column, or a Chirobiotic $\mathrm{T}$ column. Optical rotations were determined using a polarimeter set at $589 \mathrm{~nm}$.

( \pm ) Dimethyl [1-hydroxy-2-propenyl] phosphonate 1a. To a mixture of dimethyl phosphite (27.5 mL, $300 \mathrm{mmol})$ and acrolein $(24 \mathrm{~mL}, 360 \mathrm{mmol})$ was added $\mathrm{Et}_{3} \mathrm{~N}(21 \mathrm{~mL}, 150 \mathrm{mmol})$. The reaction mixture was stirred overnight and then the excess acrolein and $\mathrm{Et}_{3} \mathrm{~N}$ were evaporated in vacuo to give hydroxy phosphonate $1 \mathbf{a}$ in quantative yield. IR (neat) $3288 \mathrm{~cm}^{-1} ;{ }^{1} \mathrm{H}$ $\operatorname{NMR}\left(\mathrm{CDCl}_{3}\right) \delta 5.95(1 \mathrm{H}, \mathrm{m}), 5.47(1 \mathrm{H}, \mathrm{m}), 5.27(1 \mathrm{H}, \mathrm{m}), 4.88(1 \mathrm{H}$, brd S $), 3.77\left(6 \mathrm{H}, \mathrm{d}, J_{\mathrm{HP}}=\right.$ $11 \mathrm{~Hz}) ;{ }^{13} \mathrm{C} \mathrm{NMR}\left(\mathrm{CDCl}_{3}\right) \delta 132.7\left(\mathrm{~d}, J_{\mathrm{CP}}=3.4 \mathrm{~Hz}\right), 117.4\left(\mathrm{~d}, J_{\mathrm{CP}}=12 \mathrm{~Hz}\right), 69.3\left(\mathrm{~d}, J_{\mathrm{CP}}=160\right.$ $\mathrm{Hz}), 53.9\left(\mathrm{~d}, J_{\mathrm{CP}}=7 \mathrm{~Hz}\right), 53.7\left(\mathrm{~d}, J_{\mathrm{CP}}=7 \mathrm{~Hz}\right) ;{ }^{31} \mathrm{P} \mathrm{NMR}\left(\mathrm{CDCl}_{3}\right) \delta 24.5 ; \mathrm{HRMS}\left(\mathrm{EI}, \mathrm{M}^{+}\right)$calcd. for $\mathrm{C}_{5} \mathrm{H}_{12} \mathrm{O}_{4} \mathrm{P}:$ 167.0473. Found 167.0476.

(1R) Dimethyl [1-hydroxy-2-propenyl] phosphonate $(R) 1$. To a solution of anhydrous dimethyl (L)-tartrate $(3.24 \mathrm{~g}, 18.2 \mathrm{mmol})$ in freshly distilled THF (140 mL) was added distilled $\mathrm{Ti}(\mathrm{O} i \mathrm{Pr})_{4}(5.17 \mathrm{~g}, 18.2 \mathrm{mmol})$. The mixture was stirred at $-15^{\circ} \mathrm{C}$ for 30 minutes to insure complete complexation. Acrolein $(5.1 \mathrm{~g}, 91 \mathrm{mmol})$ was added and the mixture was stirred for an additional 15 minutes. Dimethyl phosphite (20 g, $182 \mathrm{mmol})$ was added and the reaction mixture was placed in the freezer (approx. $-15^{\circ} \mathrm{C}$ ) overnight. The reaction mixture was removed from the freezer and chelex ${ }^{\circledR}$ (approx 57g) was added. The mixture was stirred at r.t. for 2 days. The chelex ${ }^{\circledR}$ was removed by filtration and washed several times with $\mathrm{CH}_{2} \mathrm{Cl}_{2}$. The filtrate was concentrated and placed on a vacuum line until all dimethyl phosphite had evaporated $\left({ }^{31} \mathrm{P}\right.$ NMR). The crude product was purified by chromatography $\left(\mathrm{SiO}_{2}, \mathrm{EtOAc}\right)$ to give the hydroxy phosphonate 1a (11.8 g, 78\%). The enantiomeric excess was determined by ${ }^{31} \mathrm{P}$ NMR spectroscopy (Karfarski's quinine method) ${ }^{1}$ to be $70 \%$. 
Dimethyl [1-(methoxycarbonyloxy)-2-propenyl] phosphonate 3a. Hydroxy phosphonate 1a (4.78 g, $28.8 \mathrm{mmol}$ ) was dissolved in anhydrous $\mathrm{CH}_{2} \mathrm{Cl}_{2}(40 \mathrm{~mL})$ and the solution was cooled to $0^{\circ} \mathrm{C}$. Pyridine $(3.7 \mathrm{~mL}, 43.2 \mathrm{mmol})$ and DMAP $(0.049 \mathrm{~g}, 0.40 \mathrm{mmol})$ were added to the reaction, followed by the slow addition of methyl chloroformate $(4.9 \mathrm{~mL}, 63.3 \mathrm{mmol})$. After addition of methyl chloroformate was complete, the reaction mixture was allowed to warm to room temperature and then it was stirred until the reaction was complete (TLC, 24hr). The reaction mixture was washed with $\mathrm{H}_{2} \mathrm{O}(2 \mathrm{x})$ and saturated $\mathrm{CuSO}_{4}(2 \mathrm{x})$, and then the organic layer was dried over anhydrous $\mathrm{MgSO}_{4}$. The solvent was evaporated in vacuo and the crude product was purified by chromatography $\left(\mathrm{SiO}_{2}\right.$, EtOAc, $\left.100 \%\right)$ to give 3a as a colorless oil (5.25 g, 81\%). IR (neat) $1756.5 \mathrm{~cm}^{-1} ;{ }^{1} \mathrm{H} \mathrm{NMR}\left(\mathrm{CDCl}_{3}\right) \delta 5.79(1 \mathrm{H}, \mathrm{m}), 5.36(1 \mathrm{H}, \mathrm{m}), 5.31(1 \mathrm{H}, \mathrm{m}), 5.24(1 \mathrm{H}, \mathrm{m})$, $3.66(3 \mathrm{H}, \mathrm{s}), 3.65\left(6 \mathrm{H}, \mathrm{d}, J_{\mathrm{HP}}=12 \mathrm{~Hz}\right) ;{ }^{13} \mathrm{C} \mathrm{NMR}\left(\mathrm{CDCl}_{3}\right) \delta 154.5\left(\mathrm{~d}, J_{\mathrm{CP}}=9.3 \mathrm{~Hz}\right), 128.7\left(\mathrm{~d}, J_{\mathrm{CP}}\right.$ $=4.1 \mathrm{~Hz}), 119.5\left(\mathrm{~d}, J_{\mathrm{CP}}=11 \mathrm{~Hz}\right), 72.7\left(\mathrm{~d}, J_{\mathrm{CP}}=169 \mathrm{~Hz}\right) 55.2,53.8\left(\mathrm{~d}, J_{\mathrm{CP}}=6.9 \mathrm{~Hz}\right), 53.5\left(\mathrm{~d}, J_{\mathrm{CP}}\right.$ $=6.5 \mathrm{~Hz}) ;{ }^{31} \mathrm{P}$ NMR $\left(\mathrm{CDCl}_{3}\right) \delta 19.5 ; \operatorname{HRMS}\left(\mathrm{EI}, \mathrm{M}^{+}\right)$calcd. for $\mathrm{C}_{7} \mathrm{H}_{13} \mathrm{O}_{6} \mathrm{P}: 224.0450$. Found 224.0444 .

(1R) (2E) Dimethyl (1-hydroxy-3-phenyl-2-propenyl) phosphonate $(R) 1 \mathrm{~b} .{ }^{2}$ To a solution of dimethyl (L)-tartrate $(2.79 \mathrm{~g}, 15.25 \mathrm{mmol})$ in freshly distilled diethyl ether $(130 \mathrm{~mL})$ was added distilled $\operatorname{Ti}(\mathrm{O} i \mathrm{Pr})_{4}(4.5 \mathrm{~mL}, 15.2 \mathrm{mmol})$. The mixture was stirred at $-15^{\circ} \mathrm{C}$ for 30 minutes to insure complete complexation. Cinnamaldehyde $(9.54 \mathrm{~mL}, 76.2 \mathrm{mmol})$ was added and the mixture was stirred for an additional 15 minutes. Dimethyl phosphite (10.5 g, $114 \mathrm{mmol})$ was added and the reaction mixture was placed in the freezer (approx. $-15^{\circ} \mathrm{C}$ ). After the reaction was complete (TLC, $\mathrm{SiO}_{2}, \mathrm{EtOAc}$ ), the reaction mixture was quenched with deionized $\mathrm{H}_{2} \mathrm{O}$ and extracted with $\mathrm{CH}_{2} \mathrm{Cl}_{2}$. The combined extracts were dried over $\mathrm{Na}_{2} \mathrm{SO}_{4}$ and evaporated in vacuo to give crude product ( $15.6 \mathrm{~g}, 85 \% \mathrm{y}, 70 \%$ e.e.). The crude product was dissolved in hot EtOAc 
and cooled to give an initial crystalline fraction $(5.2 \mathrm{~g}, 28 \%)$ with an e.e. of $42 \%$. The mother liquor (e.e. 89\%) was decanted, concentrated and recooled to give an additional batch of crystals $(5.6 \mathrm{~g}, 30 \%)$ with an e.e. of $98 \%$. The remaining mother liquor was evaporated to dryness and purified by chromatography $\left(\mathrm{SiO}_{2}\right.$, EtOAc) to give a third batch of phosphonate $(2.2 \mathrm{~g}, 12 \%)$ with an e.e. of $79 \%$ (total yield $13 \mathrm{~g}, 71 \%)$. ${ }^{1} \mathrm{H}$ NMR $\left(\mathrm{CDCl}_{3}\right) \delta 7.40(2 \mathrm{H}, \mathrm{m}), 7.28(3 \mathrm{H}, \mathrm{m}), 6.80$ $\left(1 \mathrm{H}, \mathrm{ddd}, J_{\mathrm{HH}}=15,1.5 \mathrm{~Hz}, J_{\mathrm{HP}}=4.9 \mathrm{~Hz}\right), 6.35\left(1 \mathrm{H}, \mathrm{ddd}, J_{\mathrm{HH}}=16,6.2 \mathrm{~Hz}, J_{\mathrm{HP}}=5.6 \mathrm{~Hz}\right), 4.73$ $\left(1 \mathrm{H}, \mathrm{ddd}, J_{\mathrm{HH}}=6.2,1.6 \mathrm{~Hz}, J_{\mathrm{HP}}=13 \mathrm{~Hz}\right), 3.85\left(3 \mathrm{H}, \mathrm{d}, J_{\mathrm{HP}}=10 \mathrm{~Hz}\right), 3.81\left(3 \mathrm{H}, \mathrm{d}, J_{\mathrm{HP}}=10 \mathrm{~Hz}\right)$; ${ }^{13} \mathrm{C} \mathrm{NMR}\left(\mathrm{CDCl}_{3}\right) \delta 136.1\left(\mathrm{~d}, J_{\mathrm{CP}}=2.9 \mathrm{~Hz}\right), 132.2\left(\mathrm{~d}, J_{\mathrm{CP}}=13 \mathrm{~Hz}\right), 128.4,127.8,126.5,123.5$ $\left(\mathrm{d}, J_{\mathrm{CP}}=4.3 \mathrm{~Hz}\right), 69.2\left(\mathrm{~d}, J_{\mathrm{CP}}=161 \mathrm{~Hz}\right), 53.9\left(\mathrm{~d}, J_{\mathrm{CP}}=7.1 \mathrm{~Hz}\right), 53.7\left(\mathrm{~d}, J_{\mathrm{CP}}=7.4 \mathrm{~Hz}\right) ;{ }^{31} \mathrm{P}$ NMR $\left(\mathrm{CDCl}_{3}\right) \delta 23.8$.

(2E) Dimethyl [1-(methoxycarbonyloxy)-3-phenyl-2-propenyl] phosphonate 3b. Hydroxy phosphonate $1 \mathbf{b}(2.0 \mathrm{~g}, 8.26 \mathrm{mmol})$ was dissolved in anhydrous $\mathrm{CH}_{2} \mathrm{Cl}_{2}(30 \mathrm{~mL})$ and the solution was cooled to $0^{\circ} \mathrm{C}$. Pyridine $(1 \mathrm{~mL}, 12.4 \mathrm{mmol})$ and DMAP $(0.014 \mathrm{~g}, 0.11 \mathrm{mmol})$ were added, followed by the slow addition of methyl chloroformate $(1.3 \mathrm{~mL}, 16.5 \mathrm{mmol})$. After addition of methyl chloroformate was complete, the reaction mixture was allowed to warm to room temperature and then it was stirred until the reaction was complete (TLC). The reaction mixture was washed with $\mathrm{H}_{2} \mathrm{O}(2 \mathrm{x})$ and saturated $\mathrm{CuSO}_{4}(2 \mathrm{x})$, then the organic layer was dried over anhydrous $\mathrm{MgSO}_{4}$. The solvent was evaporated in vacuo and the crude product was purified by chromatography $\left(\mathrm{SiO}_{2}\right.$, EtOAc, 100\%) to give phosphonate $\mathbf{3 b}$ as a colorless oil $(2.35 \mathrm{~g}, 95 \%)$. IR (neat) $1755.1 \mathrm{~cm}^{-1} ;{ }^{1} \mathrm{H} \mathrm{NMR}\left(\mathrm{CDCl}_{3}\right) \delta 7.21(2 \mathrm{H}, \mathrm{m}), 7.09(3 \mathrm{H}, \mathrm{m}), 6.59\left(1 \mathrm{H}\right.$, app. dd, $J_{\mathrm{HH}}=$ $\left.16 \mathrm{~Hz}, J_{\mathrm{HP}}=4.0 \mathrm{~Hz}\right), 6.04(1 \mathrm{H}, \mathrm{m}), 5.45\left(1 \mathrm{H}, \mathrm{ddd}, J_{\mathrm{HH}}=1.2,7.4 \mathrm{~Hz}, J_{\mathrm{HP}}=14 \mathrm{~Hz}\right), 3.61(6 \mathrm{H}, \mathrm{d}$, $\left.J_{\mathrm{HP}}=11 \mathrm{~Hz}\right), 3.62(3 \mathrm{H}, \mathrm{s}) ;{ }^{13} \mathrm{C} \mathrm{NMR}\left(\mathrm{CDCl}_{3}\right) \delta 154.9\left(\mathrm{~d}, J_{\mathrm{CP}}=9.7 \mathrm{~Hz}\right), 135.8\left(\mathrm{~d}, J_{\mathrm{CP}}=12 \mathrm{~Hz}\right)$, 
$135.7\left(\mathrm{~d}, J_{\mathrm{CP}}=2.2 \mathrm{~Hz}\right), 128.8,127.1\left(\mathrm{~d}, J_{\mathrm{CP}}=1.4 \mathrm{~Hz}\right), 119.5\left(\mathrm{~d}, J_{\mathrm{CP}}=4.4 \mathrm{~Hz}\right), 73.3\left(\mathrm{~d}, J_{\mathrm{CP}}=170\right.$

$\mathrm{Hz}), 55.6,54.2\left(\mathrm{~d}, J_{\mathrm{CP}}=7.0 \mathrm{~Hz}\right), 54.0\left(\mathrm{~d}, J_{\mathrm{CP}}=6.4 \mathrm{~Hz}\right) ;{ }^{31} \mathrm{P} \mathrm{NMR}\left(\mathrm{CDCl}_{3}\right) \delta 19.6$.

3-(3-methoxyphenyl)propene 6. To 3-methoxyphenylmagnesium bromide $(30 \mathrm{~mL}, 1.0 \mathrm{M}$ solution in THF) was added allyl bromide $(5.2 \mathrm{~mL}, 60 \mathrm{mmol})$ slowly. The reaction was stirred at room temperature for $24 \mathrm{~h}$. The reaction mixture was partitioned between $\mathrm{Et}_{2} \mathrm{O}$ and saturated $\mathrm{NH}_{4} \mathrm{Cl}$. After separation, the aqueous layer was re-extracted with $\mathrm{Et}_{2} \mathrm{O}$, and the combined organic layers were dried over anhydrous $\mathrm{MgSO}_{4}$ and concentrated in vacuo. The crude product was purified by chromatography $\left(\mathrm{SiO}_{2}\right.$, hexane) to give 3-(3-methoxyphenyl)propene 6 as a colorless liquid (4.4 g, 97\% yield); ${ }^{1} \mathrm{H}$ NMR $\left(\mathrm{CDCl}_{3}\right) \delta 7.25(1 \mathrm{H}, \mathrm{m}), 6.80(3 \mathrm{H}, \mathrm{m}), 6.01(1 \mathrm{H}$, m), $5.13(2 \mathrm{H}, \mathrm{m}), 3.83(3 \mathrm{H}, \mathrm{s}), 3.41(2 \mathrm{H}, \mathrm{d}, J=6.7 \mathrm{~Hz}) ;{ }^{13} \mathrm{C} \mathrm{NMR}\left(\mathrm{CDCl}_{3}\right) \delta 159.9,141.9$, $137.4,129.5,121.2,116.1,114.5,111.6,55.3,40.4$.

$(1 R)$ (2E) Dimethyl [1-(methoxycarbonyloxy)-4-(3-methoxyphenyl)-2-

butenyl]phosphonate 3d from Phosphonate 1b. $2^{\text {nd }}$ generation Grubbs catalyst $(0.211$ g, 0.249 mmol) was dissolved in $\mathrm{CH}_{2} \mathrm{Cl}_{2}(23 \mathrm{~mL})$. Phosphonate 1b $(2.35 \mathrm{~g}, 9.69 \mathrm{mmol})$ and 3-(3methoxyphenyl)propene 6 ( $2.49 \mathrm{~g}, 16.8 \mathrm{mmol})$ were added and the reaction flask was placed in a pre-heated oil bath and heated at $40^{\circ} \mathrm{C}$ for $12 \mathrm{~h}$. The reaction mixture was allowed to cool and then the solvent was evaporated in vacuo. The crude product was purified by chromatography $\left(\mathrm{SiO}_{2}, \mathrm{CH}_{2} \mathrm{Cl}_{2}\right.$ :EtOAc, 20:80) to give a mixture of phosphonate $\mathbf{1 b}$ and dimethyl [1-hydroxy-4(3-methoxyphenyl)-2-butenyl]phosphonate 3d as a pale yellow oil (1.96 g). The isolated mixture was cycled through the metathesis reaction three more times to give dimethyl [1-hydroxy-4-(3methoxyphenyl)-2-butenyl]phosphonate 3d $(1.0 \mathrm{~g}, 36 \%) .{ }^{31} \mathrm{P}$ NMR $\left(\mathrm{CDCl}_{3}\right) \delta 24.5 . \quad$ The hydroxy phosphonate $1 \mathbf{d}(1.0 \mathrm{~g}, 3.49 \mathrm{mmol})$ was dissolved in anhydrous $\mathrm{CH}_{2} \mathrm{Cl}_{2}(15 \mathrm{~mL})$ and the solution was cooled to $0^{\circ} \mathrm{C}$. Pyridine $(0.45 \mathrm{~mL}, 5.24 \mathrm{mmol})$ and DMAP $(0.006 \mathrm{~g}, 0.04 \mathrm{mmol})$ 
were added to the reaction, followed by the slow addition of methyl chloroformate $(0.7 \mathrm{~mL}, 8.47$ mmol). After addition of methyl chloroformate was complete, the reaction mixture was allowed to warm to room temperature and then it was stirred until the reaction was complete (TLC, 24hr). The reaction mixture was washed with $\mathrm{H}_{2} \mathrm{O}(2 \mathrm{x})$ and saturated $\mathrm{CuSO}_{4}(2 \mathrm{x})$, and then the organic layer was dried over anhydrous $\mathrm{MgSO}_{4}$. The solvent was evaporated in vacuo and the crude product was purified by chromatography $\left(\mathrm{SiO}_{2}\right.$, EtOAc, $\left.100 \%\right)$ to give $\mathbf{3 d}$ as a colorless oil $(1.14$ g, $95 \%)$.

A General Procedure for the Addition of Malonates to Allylic Carbonates 3b-d. NaH (3.47 mmol) was suspended in anhydrous THF $(16 \mathrm{~mL})$ and then the malonate $(3.47 \mathrm{mmol})$ in THF $(1 \mathrm{~mL})$ was added. The mixture was stirred at room temperature for 3-4 minutes. The phosphonate $(2.9 \mathrm{mmol})$ was added, followed by $\mathrm{Pd}\left(\mathrm{PPh}_{3}\right)_{4}(0.09 \mathrm{mmol}, 3 \mathrm{~mol} \%)$. The reaction flask was placed in a pre-heated oil bath and heated at $70^{\circ} \mathrm{C}$ for $1 \mathrm{~h}$. The reaction mixture was allowed to cool, and then it was partitioned between brine and $\mathrm{Et}_{2} \mathrm{O}$. After separation, the aqueous layer was re-extracted with $\mathrm{Et}_{2} \mathrm{O}$ and the combined organic layers were dried over anhydrous $\mathrm{Na}_{2} \mathrm{SO}_{4}$. The solvent was evaporated in vacuo to give the crude product.

\section{Addition of Dimethyl Malonate 10a to (1R) (2E) Dimethyl [1-(methoxycarbonyloxy)-3-}

phenyl-2-propenyl] phosphonate 3b. $\mathrm{NaH}(0.056 \mathrm{~g}, 1.4 \mathrm{mmol})$, THF (10mL), phosphonate 3b (0.28 g, $0.93 \mathrm{mmol})$, malonate 10a $(0.16 \mathrm{~mL}, 1.4 \mathrm{mmol})$ and $\mathrm{Pd}\left(\mathrm{PPh}_{3}\right)_{4}(0.032 \mathrm{~g}, 0.028 \mathrm{mmol})$. The crude compound was purified by chromatography $\left(\mathrm{SiO}_{2}\right.$, EtOAc:hexanes, 2:1) to give the $E$ isomer $11 \mathrm{a}(\boldsymbol{E})$ as a pale yellow oil $(0.249 \mathrm{~g}, 75 \%)$. IR (neat) $1737.5 \mathrm{~cm}^{-1} ;{ }^{1} \mathrm{HNMR}\left(\mathrm{CDCl}_{3}\right) \delta$ $7.20(5 \mathrm{H}, \mathrm{m}), 6.83\left(1 \mathrm{H}, \mathrm{ddd}, J_{\mathrm{HH}}=7.7,17 \mathrm{~Hz}, J_{\mathrm{HP}}=25 \mathrm{~Hz}\right), 5.59\left(1 \mathrm{H}, \mathrm{ddd}, J_{\mathrm{HH}}=1.2,17 \mathrm{~Hz}, J_{\mathrm{HP}}\right.$ $=19 \mathrm{~Hz}), 4.17(1 \mathrm{H}, \mathrm{m}), 3.82\left(1 \mathrm{H}, \mathrm{d}, J_{\mathrm{HH}}=11 \mathrm{~Hz}\right), 3.68(3 \mathrm{H}, \mathrm{s}), 3.62\left(3 \mathrm{H}, \mathrm{d}, J_{\mathrm{HP}}=11 \mathrm{~Hz}\right), 3.58$ $\left(3 \mathrm{H}, \mathrm{d}, J_{\mathrm{HP}}=11 \mathrm{~Hz}\right), 3.42(3 \mathrm{H}, \mathrm{s}) ;{ }^{13} \mathrm{C} \mathrm{NMR}\left(\mathrm{CDCl}_{3}\right) \delta 167.9,167.4,152.2\left(\mathrm{~d}, J_{\mathrm{CP}}=5.4 \mathrm{~Hz}\right)$, 
$137.9,129.1,128.4,128.0,118.1\left(\mathrm{~d}, J_{\mathrm{CP}}=186 \mathrm{~Hz}\right), 56.8,53.0,52.81,52.6\left(\mathrm{~d}, J_{\mathrm{CP}}=5.7 \mathrm{~Hz}\right), 49.9$

$\left(\mathrm{d}, J_{\mathrm{CP}}=22 \mathrm{~Hz}\right) ;{ }^{31} \mathrm{P} \mathrm{NMR}\left(\mathrm{CDCl}_{3}\right) \delta 20.7$; HRMS $\left(\mathrm{EI}, \mathrm{M}^{+}\right)$calcd. for $\mathrm{C}_{16} \mathrm{H}_{21} \mathrm{O}_{7} \mathrm{P}: 356.1025$. Found 356.1021, and a small amount of the $Z$-isomer contaminated with $\mathrm{Ph}_{3} \mathrm{PO}(0.0453 \mathrm{~g}, 13 \%)$ ${ }^{1} \mathrm{H}$ NMR $7.25(5 \mathrm{H}, \mathrm{m}), 6.69\left(1 \mathrm{H}, \mathrm{ddd}, J_{\mathrm{HH}}=10,13 \mathrm{~Hz}, J_{\mathrm{HP}}=49 \mathrm{~Hz}\right), 5.55\left(1 \mathrm{H}, \mathrm{dd}, J_{\mathrm{HH}}=13 \mathrm{~Hz}\right.$, $\left.J_{\mathrm{HP}}=17 \mathrm{~Hz}\right), 5.11\left(1 \mathrm{H}\right.$, app. t, $\left.J_{\mathrm{HH}}=10 \mathrm{~Hz}\right), 3.81\left(1 \mathrm{H}, \mathrm{d}, J_{\mathrm{HH}}=10 \mathrm{~Hz}\right), 3.65(3 \mathrm{H}, \mathrm{s}), 3.64(3 \mathrm{H}, \mathrm{d}$, $\left.J_{\mathrm{HP}}=11 \mathrm{~Hz}\right), 3.54\left(3 \mathrm{H}, \mathrm{d}, J_{\mathrm{HP}}=11 \mathrm{~Hz}\right), 3.47(3 \mathrm{H}, \mathrm{s}) ;{ }^{31} \mathrm{P} \mathrm{NMR} 18.9$.

Addition of Dimethyl 2-(3-methoxyphenyl) Malonate 10b to (1R) (2E) Dimethyl [1(methoxycarbonyloxy)-3-phenyl-2-propenyl] phosphonate 3b. $\mathrm{NaH}(0.038 \mathrm{~g}, 1.59 \mathrm{mmol})$ in THF $(8 \mathrm{~mL})$, malonate $\mathbf{1 0 b}(0.0 .4 \mathrm{~g}, 1.59 \mathrm{mmol})$ in THF $(1 \mathrm{~mL})$, phosphonate $\mathbf{3 b}(0.24 \mathrm{~g}, 0.79$ $\mathrm{mmol})$, and $\mathrm{Pd}\left(\mathrm{PPh}_{3}\right)_{4}(0.018 \mathrm{~g}, 0.016 \mathrm{mmol})$ gave the malonate adduct $\mathbf{1 1 b}$ as a colorless oil (0.17 g, 45\%). ${ }^{1} \mathrm{H}$ NMR $\delta 7.30(3 \mathrm{H}, \mathrm{m}), 7.22(1 \mathrm{H}, \mathrm{m}), 7.08(3 \mathrm{H}, \mathrm{m}), 6.71(1 \mathrm{H}, \mathrm{m}), 6.60(2 \mathrm{H}, \mathrm{m})$, $5.37(1 \mathrm{H}, \mathrm{ddd}, J=1.2,17,20 \mathrm{~Hz}), 4.21(1 \mathrm{H}, \mathrm{m}), 3.72(3 \mathrm{H}, \mathrm{s}), 3.70(3 \mathrm{H}, \mathrm{s}), 6.63(3 \mathrm{H}, \mathrm{d}, J=11$ $\mathrm{Hz}), 3.62(3 \mathrm{H}, \mathrm{d}, J=11 \mathrm{~Hz}), 3.52(3 \mathrm{H}, \mathrm{s}), 314,(1 \mathrm{H}, \mathrm{d}, J=14 \mathrm{~Hz}), 2.89(1 \mathrm{H}, \mathrm{d} J=14 \mathrm{~Hz}) ;{ }^{13} \mathrm{C}$ NMR $\delta 170.1,159.3,153.7\left(\mathrm{~d}, J_{\mathrm{CP}}=6.6 \mathrm{~Hz}\right), 137.3,136.6,129.6,128.5,128.7,127.9,122.5$, $117.1\left(\mathrm{~d}, J_{\mathrm{CP}}=186 \mathrm{~Hz}\right), 115.9,112.6,63.5,55.0,54.9,54.7,52.1,40.4$.

\section{Attempted Addition of tert-Butyl Methyl 2-(3-methoxyphenyl) Malonate 10c to Dimethyl} [1-(methoxycarbonyloxy)-4-(3-methoxyphenyl)-2-butenyl]phosphonate 3d. NaH (0.0275 g, $0.696 \mathrm{mmol})$ in THF $(9 \mathrm{~mL})$, malonate $\mathbf{1 0 c}(0.256 \mathrm{~mL}, 0.87 \mathrm{mmol})$, phosphonate $3 \mathbf{d}(0.2 \mathrm{~g}, 0.58$ $\mathrm{mmol})$, and $\mathrm{Pd}\left(\mathrm{PPh}_{3}\right)_{4}(0.047 \mathrm{~g}, 0.041 \mathrm{mmol})$. The crude product was purified by chromatography $\left(\mathrm{SiO}_{2}\right.$, hexane:acetone, 50:50) to give the diene 12 contaminated with $\mathrm{Ph}_{3} \mathrm{PO}$ as a yellow oil $(38 \%){ }^{1} \mathrm{HNMR}\left(\mathrm{CDCl}_{3}\right) \delta 7.26\left(1 \mathrm{H}, \mathrm{t}, J_{\mathrm{HH}}=8 \mathrm{~Hz}\right), 7.17(1 \mathrm{H}, \mathrm{m}), 7.06(2 \mathrm{H}, \mathrm{m}), 6.89$ $(2 \mathrm{H}, \mathrm{m}), 6.82(1 \mathrm{H}, \mathrm{m}) 5.90\left(1 \mathrm{H}, \mathrm{dd}, J_{\mathrm{HH}}=16 \mathrm{~Hz}, J_{\mathrm{HP}}=19 \mathrm{~Hz},\right), 3.82(3 \mathrm{H}, \mathrm{s}), 3.67\left(6 \mathrm{H}, \mathrm{d}, J_{\mathrm{HP}}=\right.$ $11 \mathrm{~Hz}) ;{ }^{13} \mathrm{C} \mathrm{NMR}\left(\mathrm{CDCl}_{3}\right) \delta 161.2,149.6\left(\mathrm{~d}, J_{\mathrm{CP}}=6.0 \mathrm{~Hz}\right), 140.4,138.6,130.8,128.7\left(\mathrm{~d}, J_{\mathrm{CP}}=\right.$ 
$27 \mathrm{~Hz}), 120.8,117.8\left(\mathrm{~d}, J_{\mathrm{CP}}=188 \mathrm{~Hz}\right), 115.8,113.0,55.7,52.5\left(\mathrm{~d}, J_{\mathrm{CP}}=5.4 \mathrm{~Hz}\right) ;{ }^{31} \mathrm{P} \mathrm{NMR}$ $\left(\mathrm{CDCl}_{3}\right) \delta 21.3$; HRMS (EI, M $\left.{ }^{+}\right)$calcd. for $\mathrm{C}_{13} \mathrm{H}_{17} \mathrm{O}_{4} \mathrm{P}: 268.0865$. Found 268.0867.

Addition of tert-Butyl Methyl 2-(3-methoxyphenyl) Malonate 10c to (2E) Dimethyl [1(methoxycarbonyloxy)-2-butenyl] phosphonate 3c. $\mathrm{NaH}(0.0389 \mathrm{~g}, 0.973 \mathrm{mmol})$ in THF (10 $\mathrm{mL})$, malonate 10c $(0.38 \mathrm{~g}, 1.3 \mathrm{mmol})$, phosphonate $3 \mathrm{c}(0.144 \mathrm{~g}, 0.65 \mathrm{mmol}){ }^{3}$ and $\mathrm{Pd}\left(\mathrm{PPh}_{3}\right)_{4}$ $(0.022 \mathrm{~g}, 0.019 \mathrm{mmol})$. The crude product was purified by chromatography $\left(\mathrm{SiO}_{2}\right.$, hexane:acetone, 50:50) to give a diastereoisomeric mixture (50:50) of malonate adducts 11c contanimated with $\mathrm{Ph}_{3} \mathrm{PO}(0.07 \mathrm{~g}, 24 \%) \mathrm{IR}$ (neat) $1719 \mathrm{~cm}^{-1} ;{ }^{1} \mathrm{H}$ NMR $\left(\mathrm{CDCl}_{3}\right) \delta 7.16(1 \mathrm{H}, \mathrm{t}$, $\left.J_{\mathrm{HH}}=7.5 \mathrm{~Hz}\right), 6.90(1 \mathrm{H}, \mathrm{m}), 6.75(3 \mathrm{H}, \mathrm{m}), 5.64(1 \mathrm{H}, \mathrm{m}), 3.77(3 \mathrm{H}, \mathrm{s}), 3.69(9 \mathrm{H}, \mathrm{m}), 3.20(2 \mathrm{H}$, m), $2.99(1 \mathrm{H}, \mathrm{m}), 1.42(4.5 \mathrm{H}, \mathrm{s}), 1.41(4.5 \mathrm{H}, \mathrm{s}), 1.17(3 \mathrm{H}, \mathrm{m}) ;{ }^{13} \mathrm{C} \mathrm{NMR}\left(\mathrm{CDCl}_{3}\right) \delta$ 170.8, 170.7, 168.7, 168.6, 159.6, $155.3\left(\mathrm{~d}, J_{\mathrm{CP}}=5.5 \mathrm{~Hz}\right), 154.7\left(\mathrm{~d}, J_{\mathrm{CP}}=5.2 \mathrm{~Hz}\right), 137.8,129.3,129.2,122.8$, 122.7, $117.1\left(\mathrm{~d}, J_{\mathrm{CP}}=187 \mathrm{~Hz}\right), 116.5\left(\mathrm{~d}, J_{\mathrm{CP}}=187 \mathrm{~Hz}\right), 116.2,116.15,112.6,112.5,82.8,82.7$, 62.9, 62.9, 55.3, $52.5(\mathrm{~m}), 52.1,52.0,41.8\left(\mathrm{~d}, J_{\mathrm{CP}}=22 \mathrm{~Hz}\right), 41.2\left(\mathrm{~d}, J_{\mathrm{CP}}=22 \mathrm{~Hz}\right), 39.8,39.5$, 28.1, $16.1\left(\mathrm{~d}, J_{\mathrm{CP}}=1.3 \mathrm{~Hz}\right), 15.4\left(\mathrm{~d}, J_{\mathrm{CP}}=0.9 \mathrm{~Hz}\right) ;{ }^{31} \mathrm{P} \mathrm{NMR}\left(\mathrm{CDCl}_{3}\right) \delta 21.8,21.5$; HRMS (EI, $\mathrm{M}^{+}$) calcd. for $\mathrm{C}_{22} \mathrm{H}_{33} \mathrm{O}_{8} \mathrm{P}: 456.1913$. Found 456.1907 .

1. Zymanczyk-Duda, E.; Skwarczynski, M.; Lejczak, B.; Karfarski, P. Tetrahedron: Asymmetry 1996, 7, 1277.

2. Rowe, B. J.; Spilling, C. D. Tetrahedron: Asymmetry 2001, 12, 1701; Blazis, V. J.; Koeller, K. J.; Spilling, C. D. J. Org. Chem. 1995, 60, 931.

3. Rowe, B. J.; Spilling, C. D. J. Org. Chem. 2003, 68, 9502 


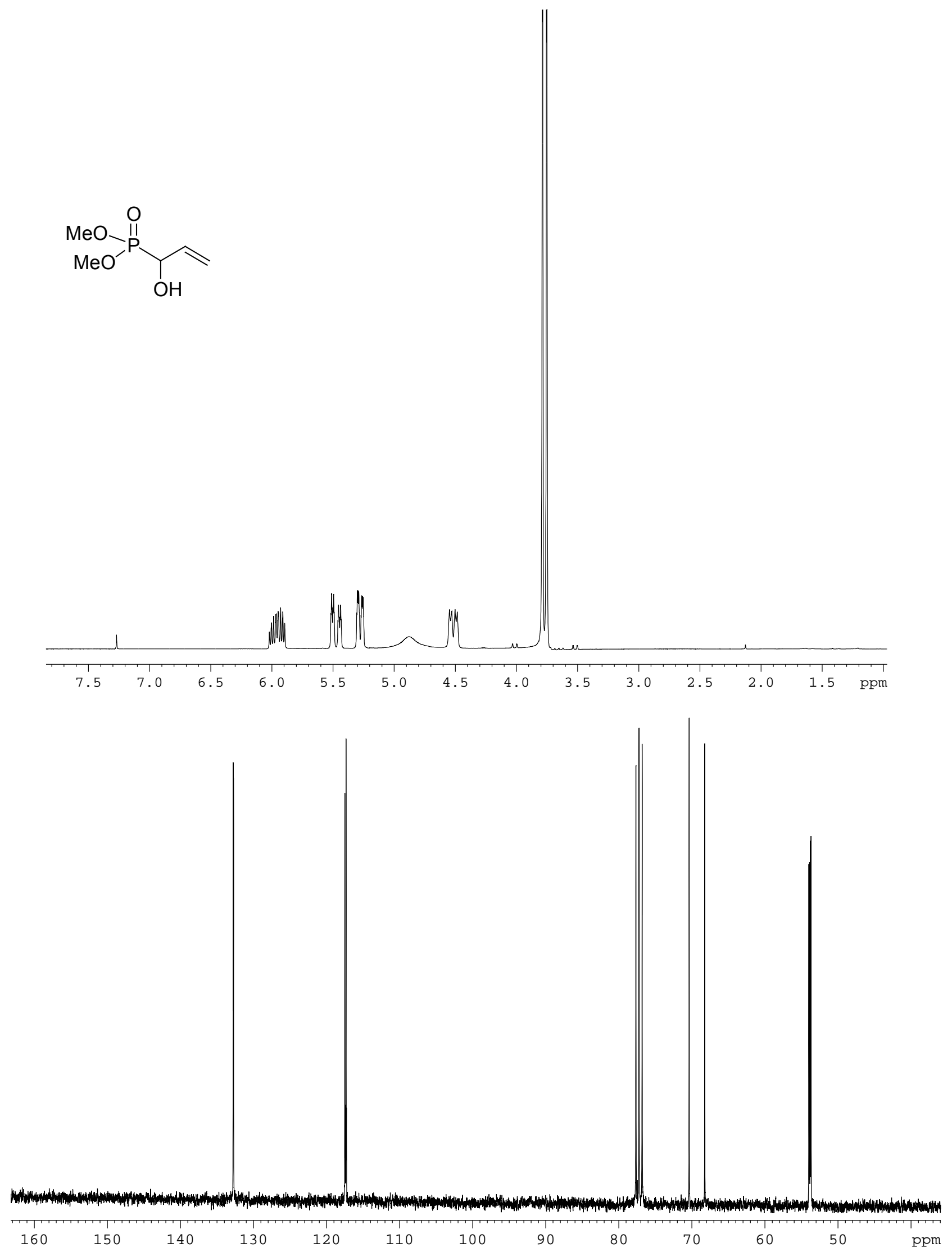




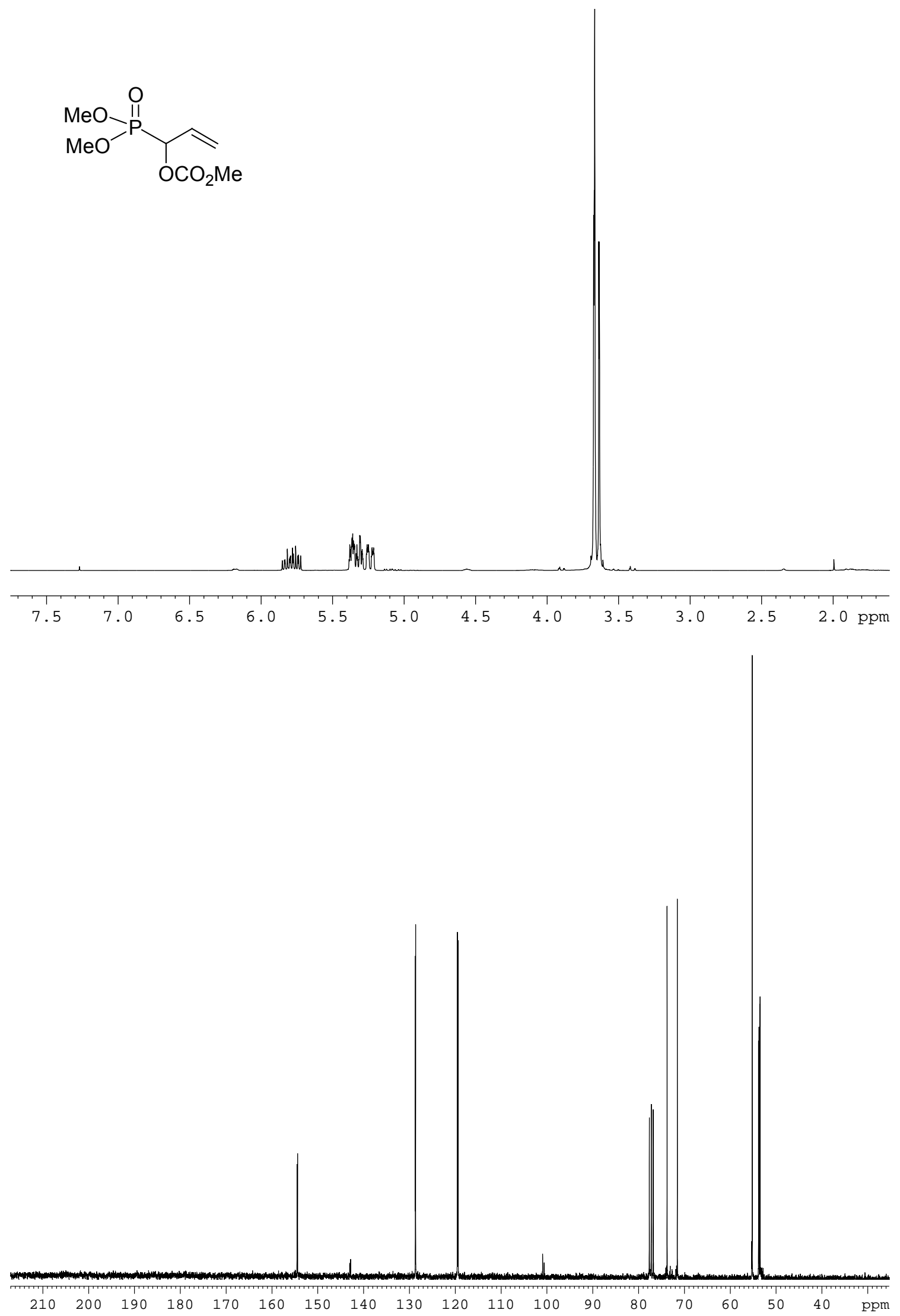


<smiles>COP(=O)(OC)C(O)/C=C/c1ccccc1</smiles>
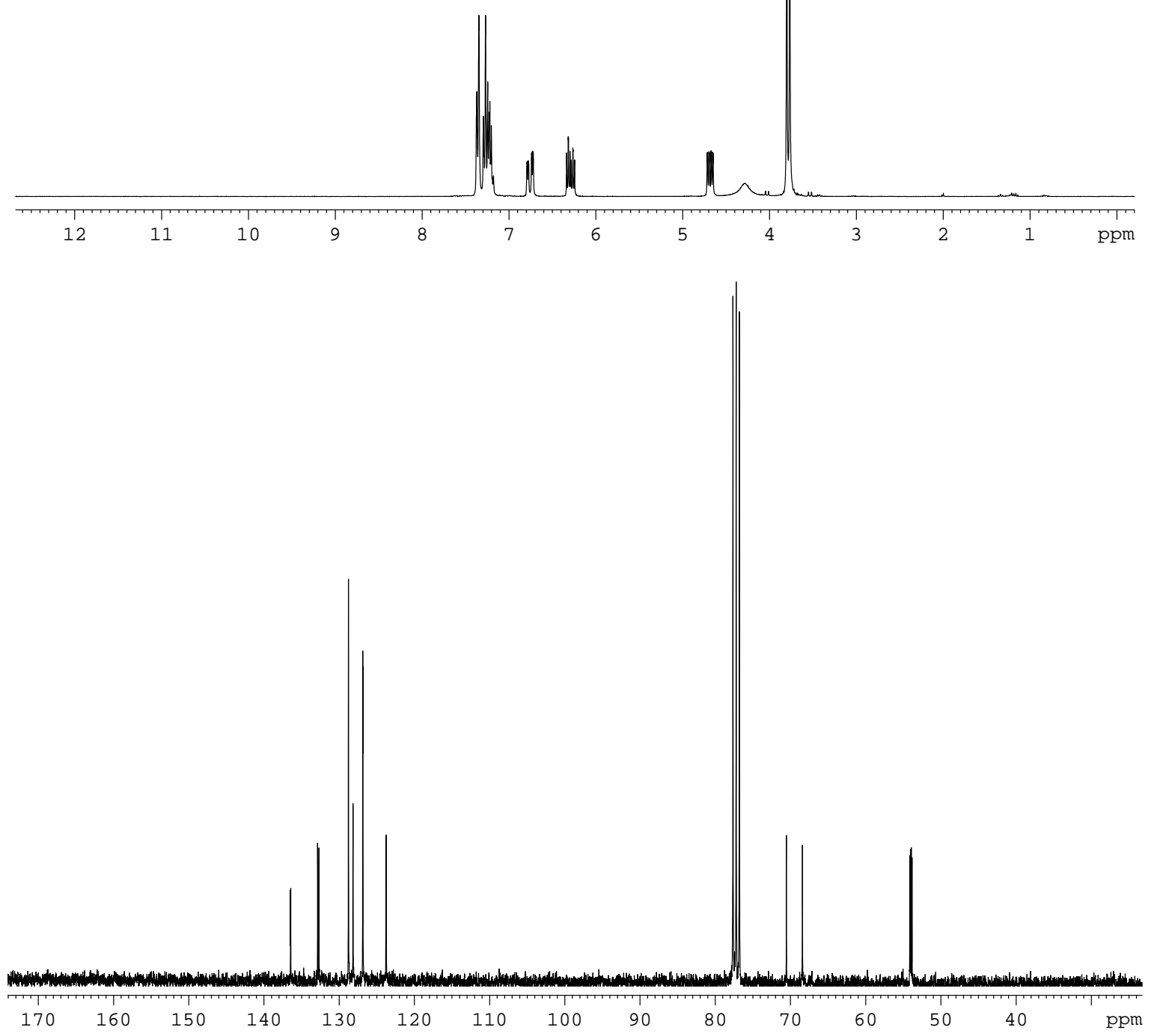

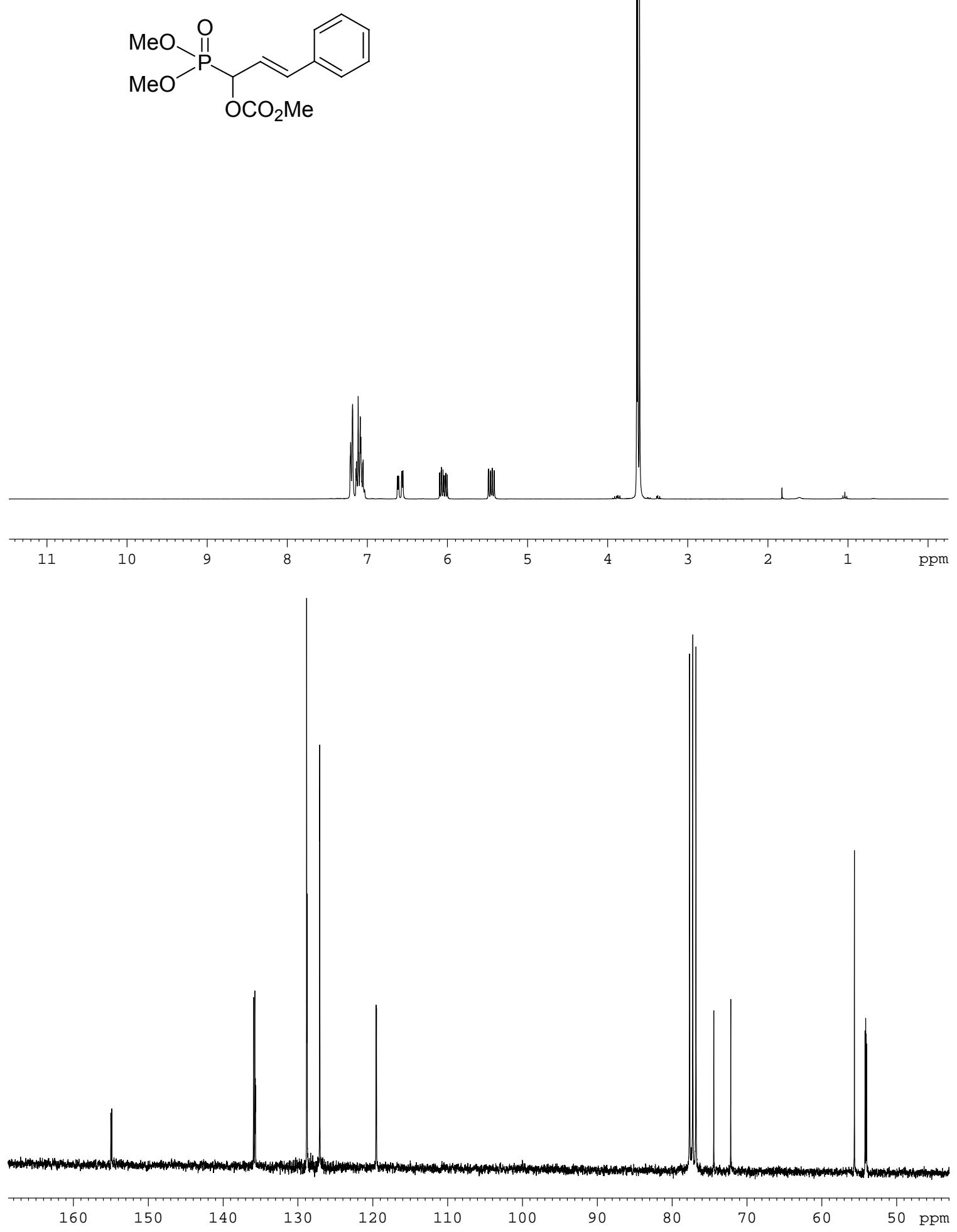


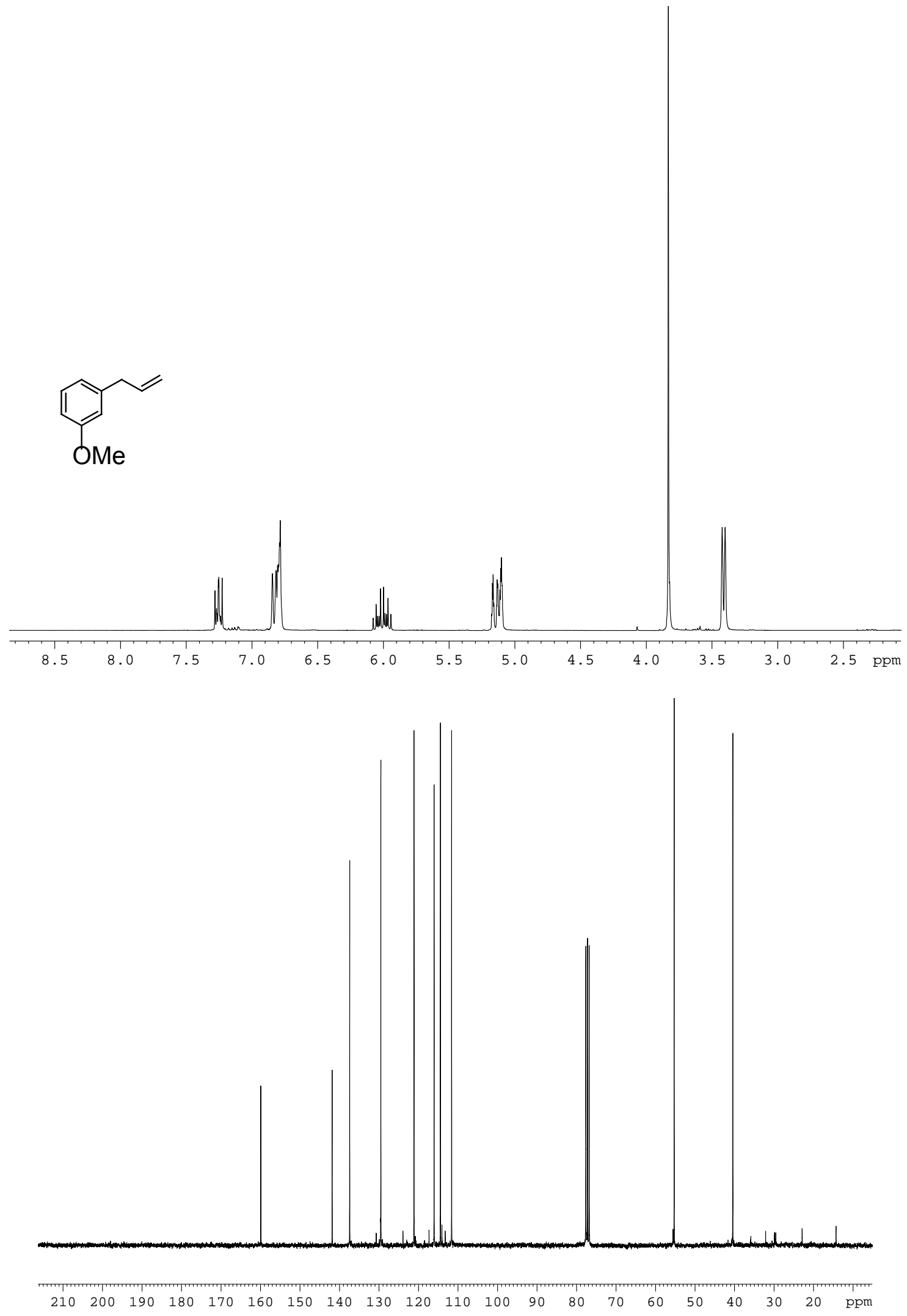



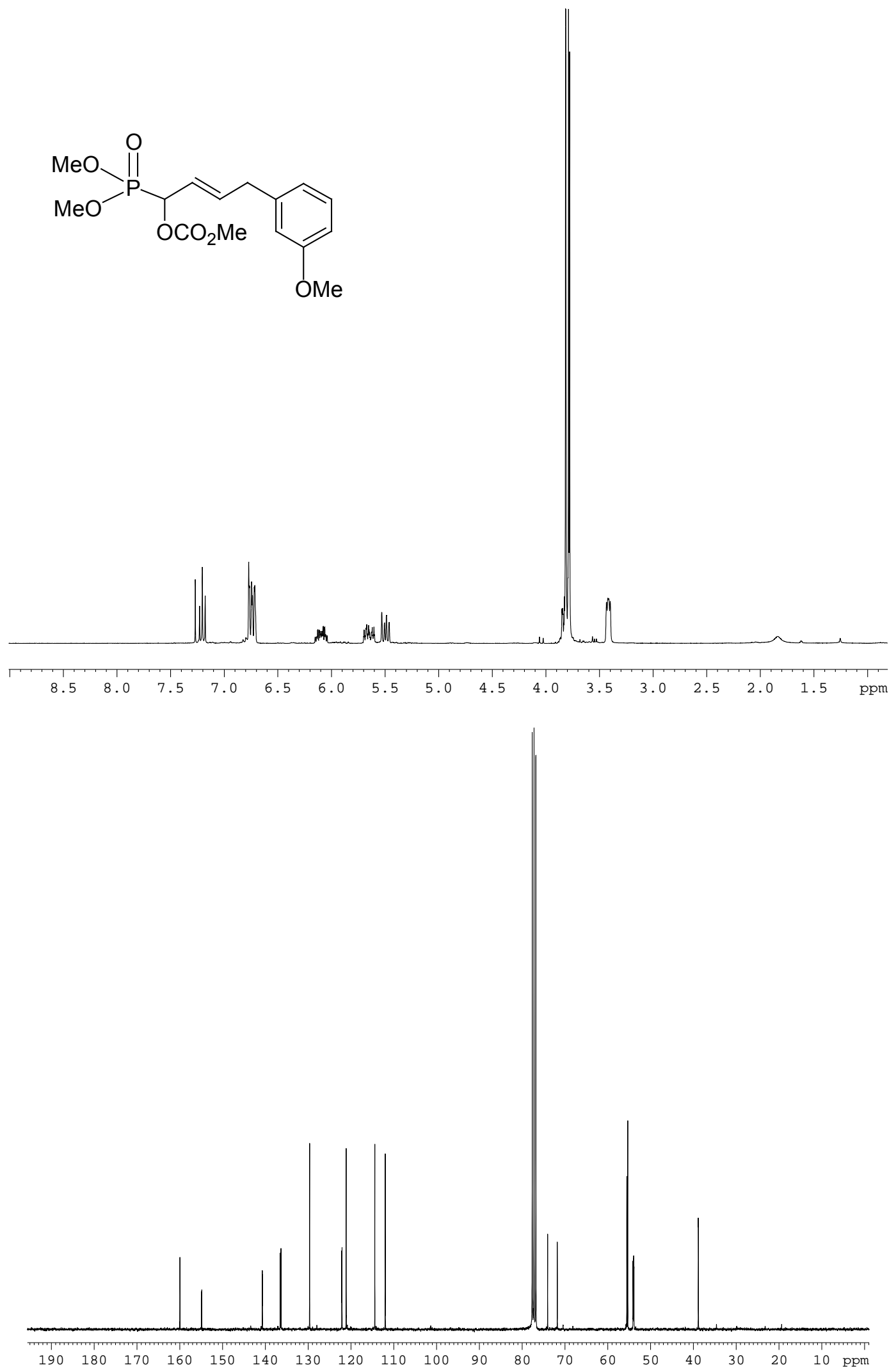


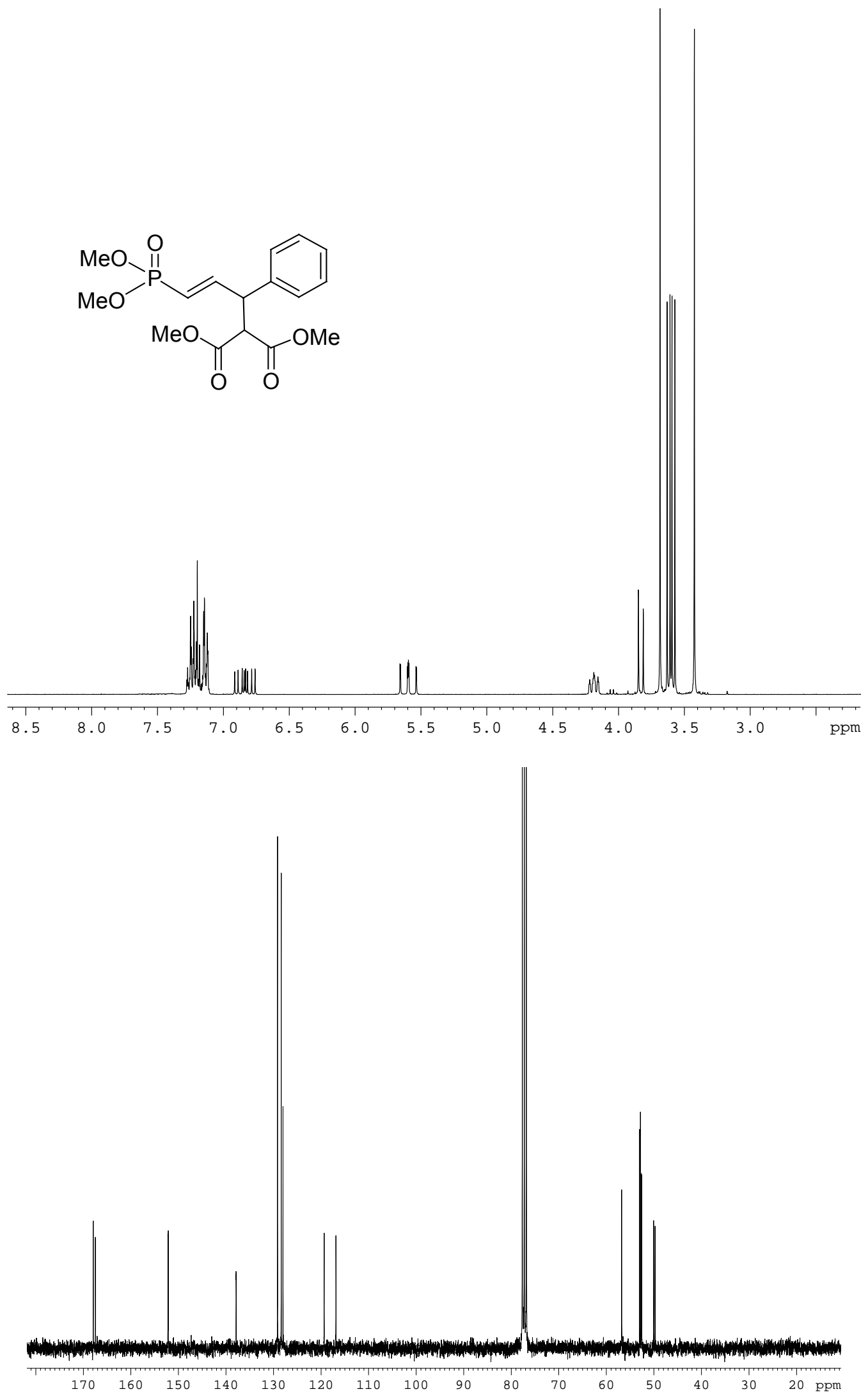




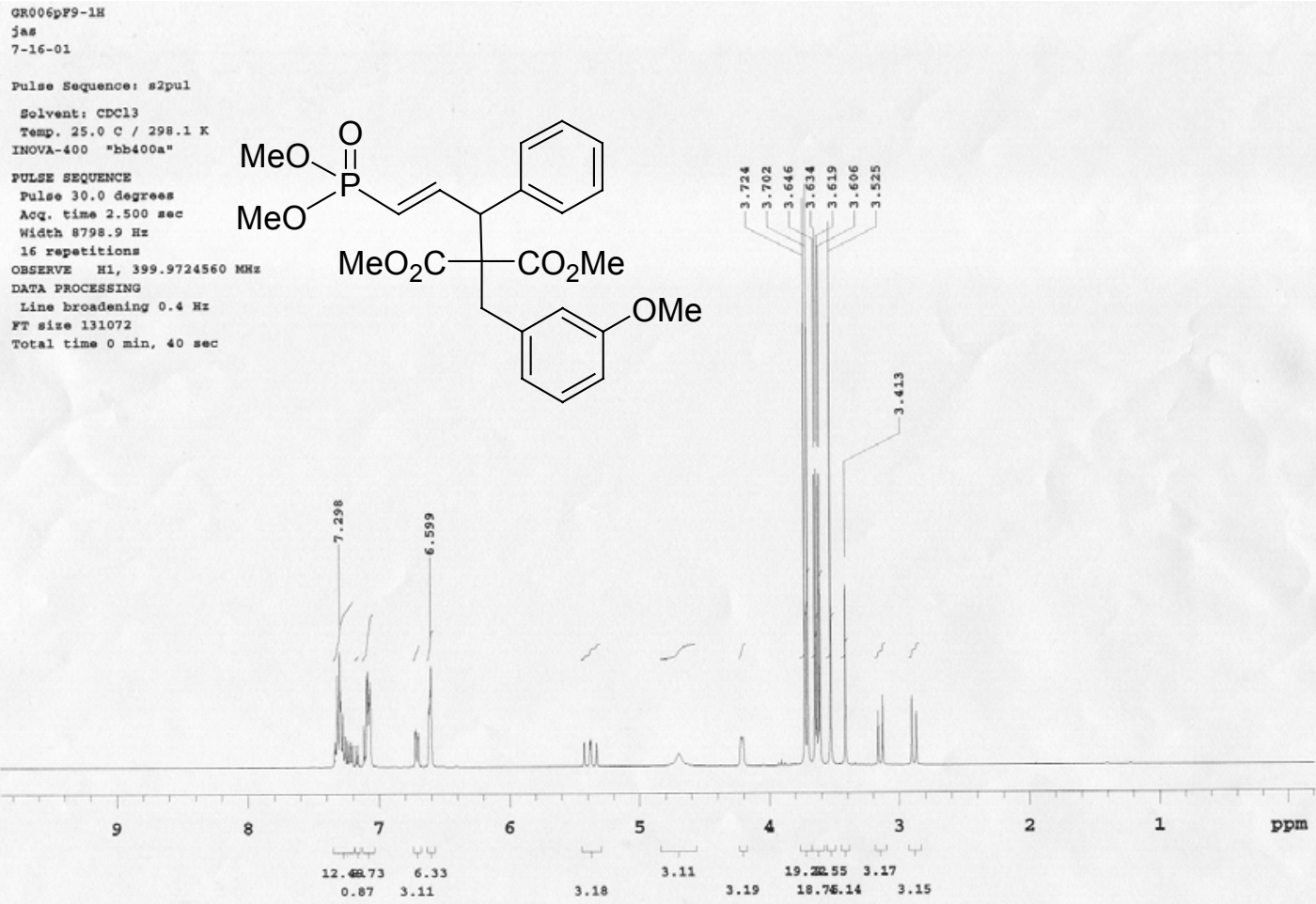

Total time 0 min, $40=0$

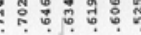

$18.76 .14 \quad 3.15$

QR006pr9-13C

jas

ํำ

Pulso sequencel s2pul

ลิ สำ ส

Tomp. 25.0 C / 298.1

Irova-400 "bb400a"

PULSE SEQUENCE

noq. timo $3.000 \mathrm{so}$

Width $24945.4 \mathrm{~Hz}$

OBSERVE C13, 100.5731544 knt

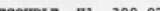

Power $44 \mathrm{~dB}$

continuounly on

Data processino

Zine bromdening $0.3 \mathrm{~g}$

Total timo $25 \mathrm{~min}, 40 \mathrm{sec}$

然

装

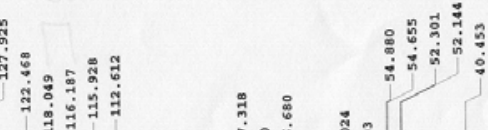

象

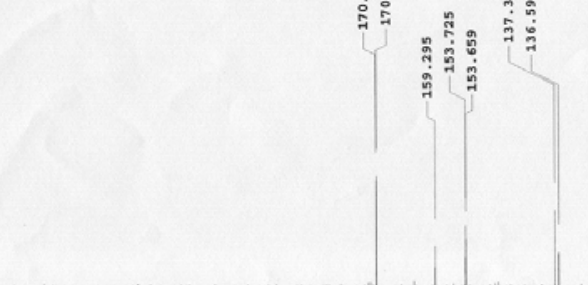

220

200

180

160

140

120

100

80

60

40

20

ppm 


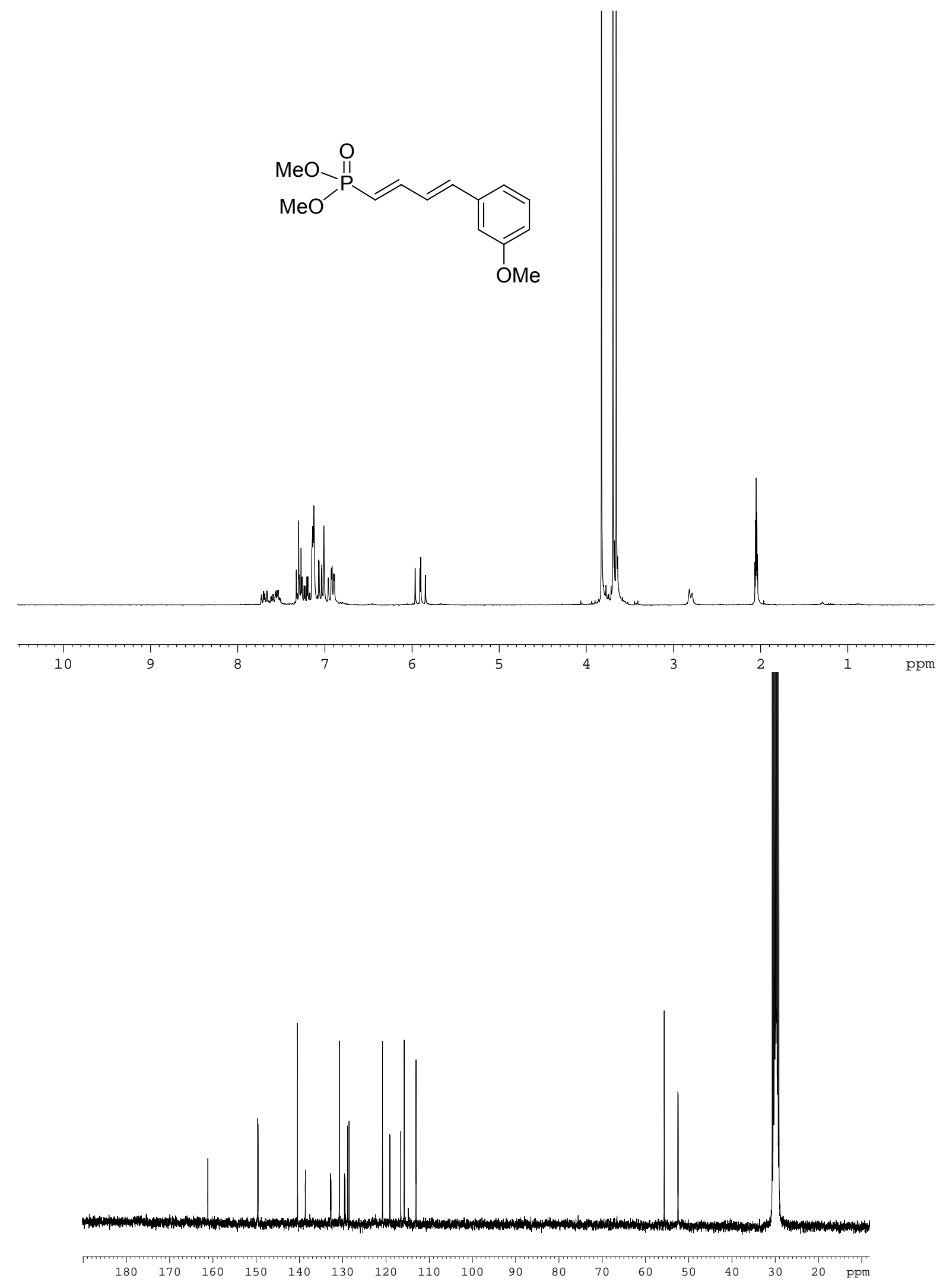



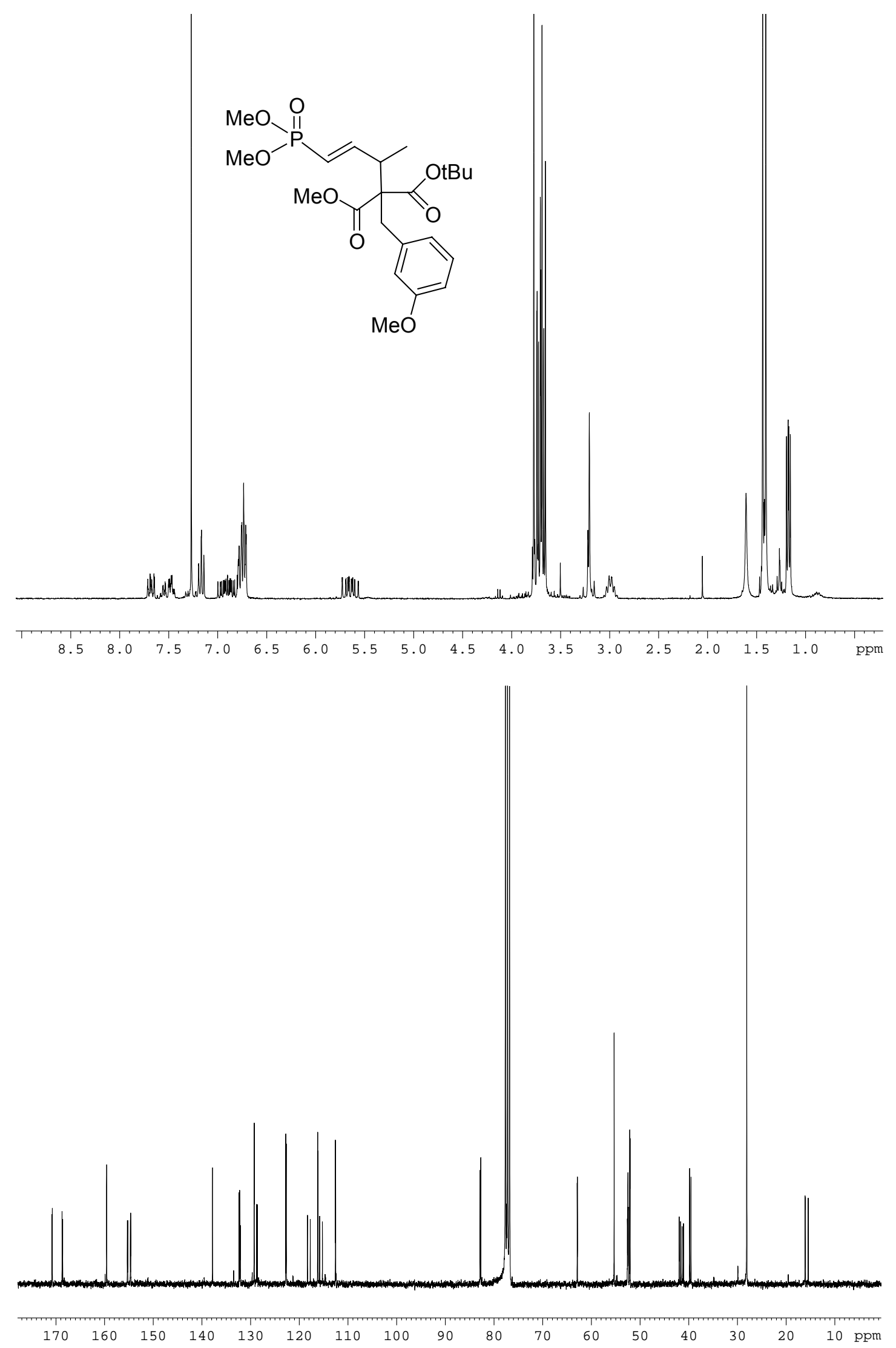


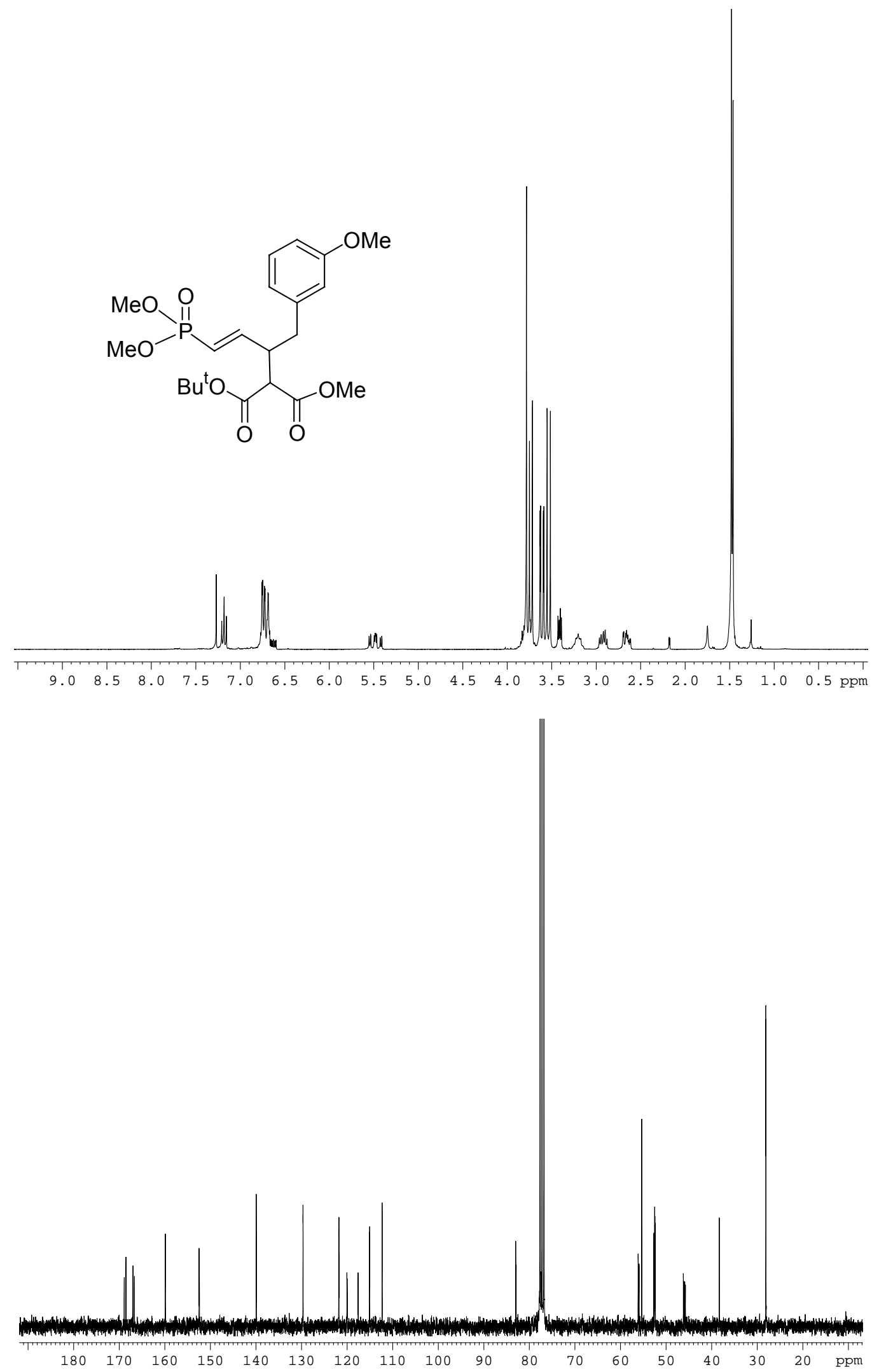




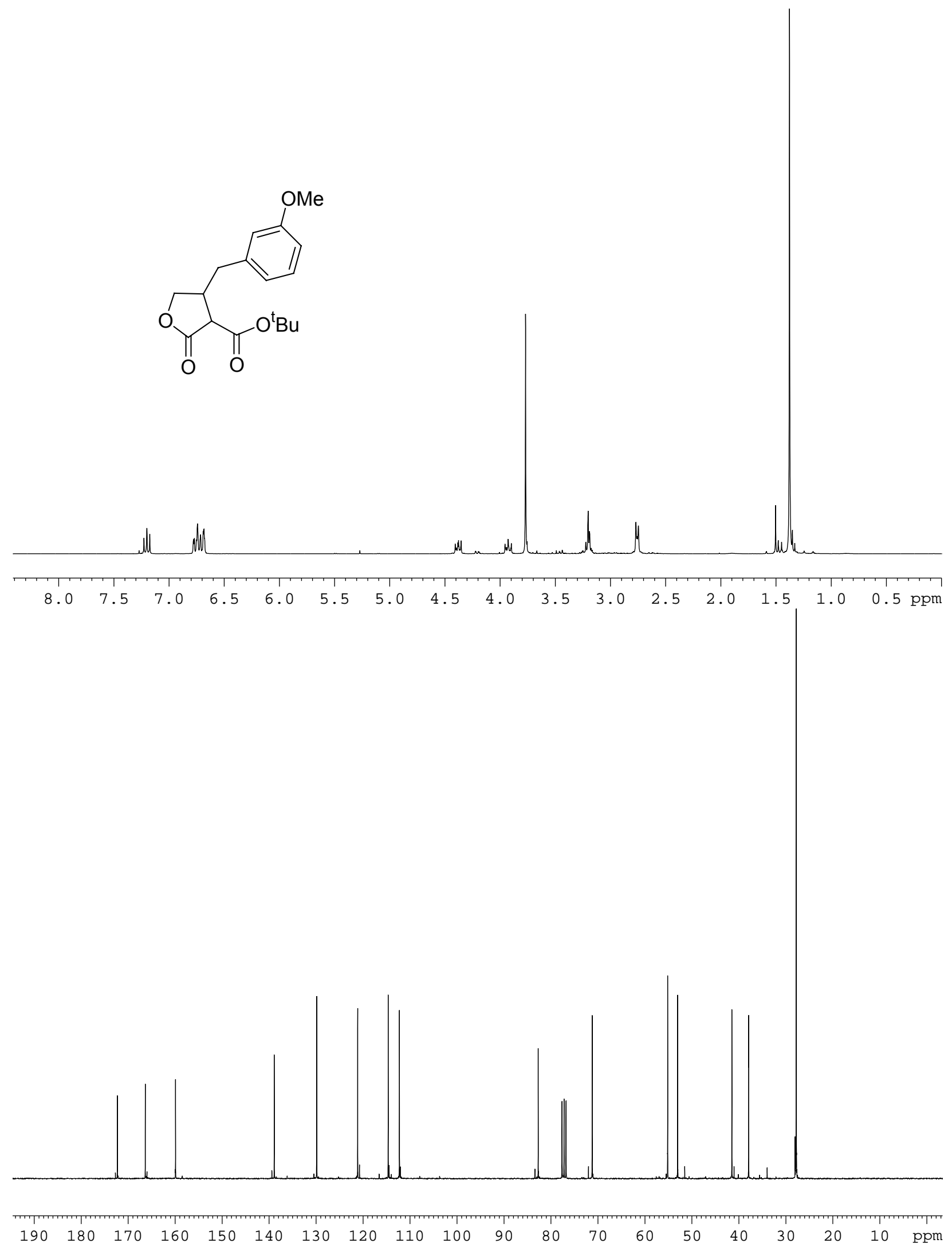



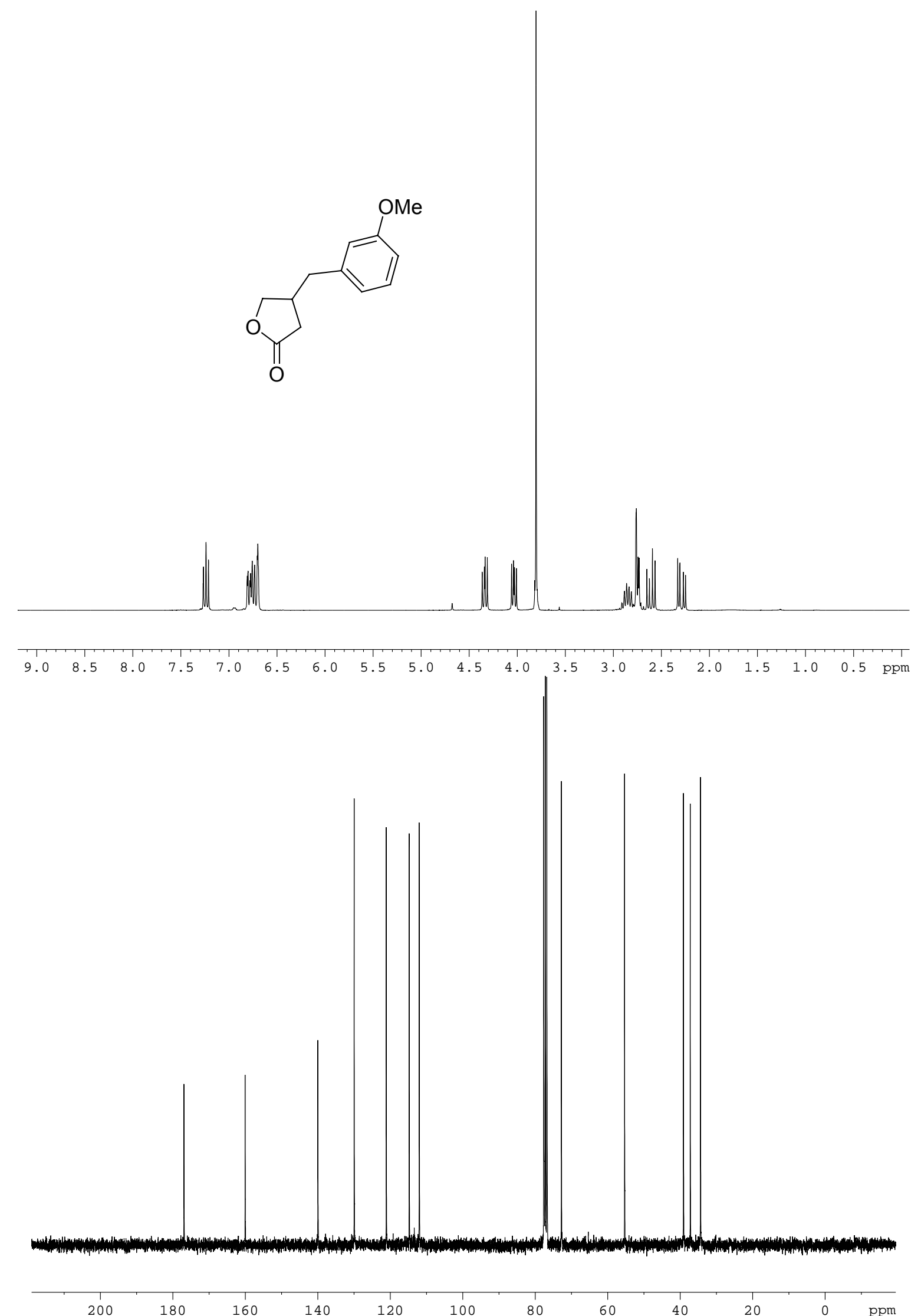
Column-S,S Whelk-O, EtOH:hexane (20:80), $1 \mathrm{~mL} / \mathrm{min}$, det $254 \mathrm{~nm}$

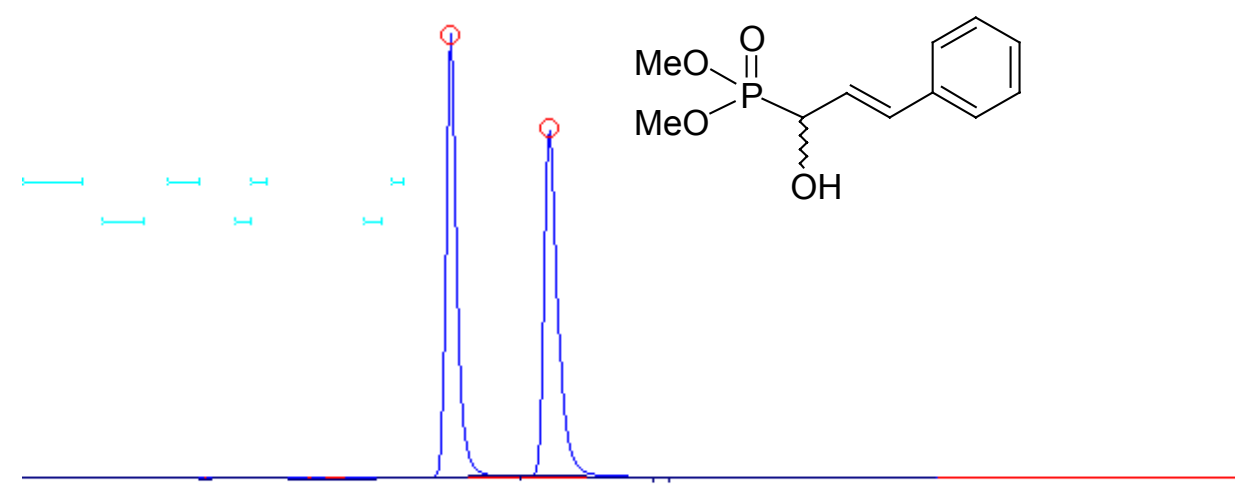

Racemic

\begin{tabular}{|c|c|c|}
\hline $\begin{array}{c}\text { Retention time } \\
(\mathrm{min})\end{array}$ & Area & \% Area \\
\hline 6.97 & 5211 & 49.7 \\
\hline 8.58 & 5281 & 50.3 \\
\hline
\end{tabular}

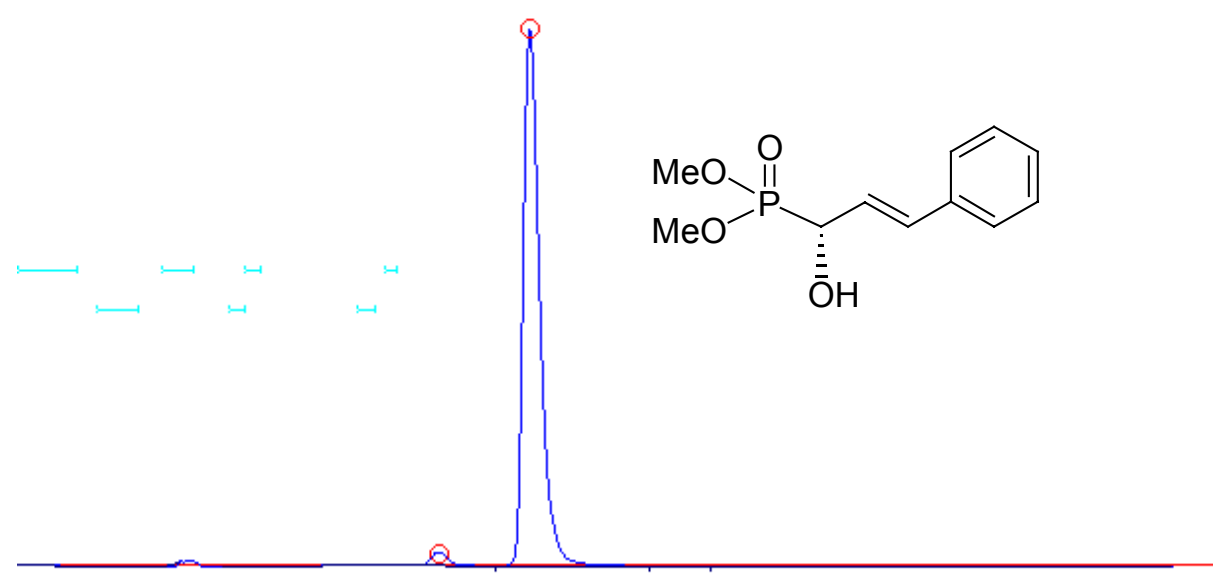

R Isomer ( $95 \%$ e.e. $)$

\begin{tabular}{|c|c|c|c|}
\hline $\begin{array}{c}\text { Retention time } \\
\text { (min) }\end{array}$ & Area & \% Area & Ratio \\
\hline 6.88 & 503 & 2.5 & 39 \\
\hline 8.38 & 19854 & 97.5 & 1 \\
\hline
\end{tabular}


<smiles>CO[C@H](/C=C/c1ccccc1)P(=O)(OC)OC</smiles>

\section{$\Lambda$}

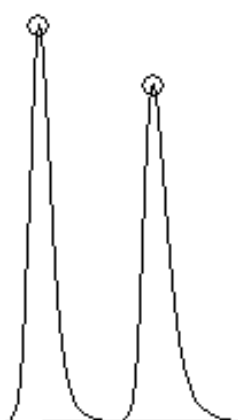

Racemic

\begin{tabular}{|c|c|c|}
\hline $\begin{array}{c}\text { Retention time } \\
(\mathrm{min})\end{array}$ & Area & $\%$ Area \\
\hline 12.65 & 5152 & 50.2 \\
\hline 14.28 & 5115 & 49.8 \\
\hline
\end{tabular}
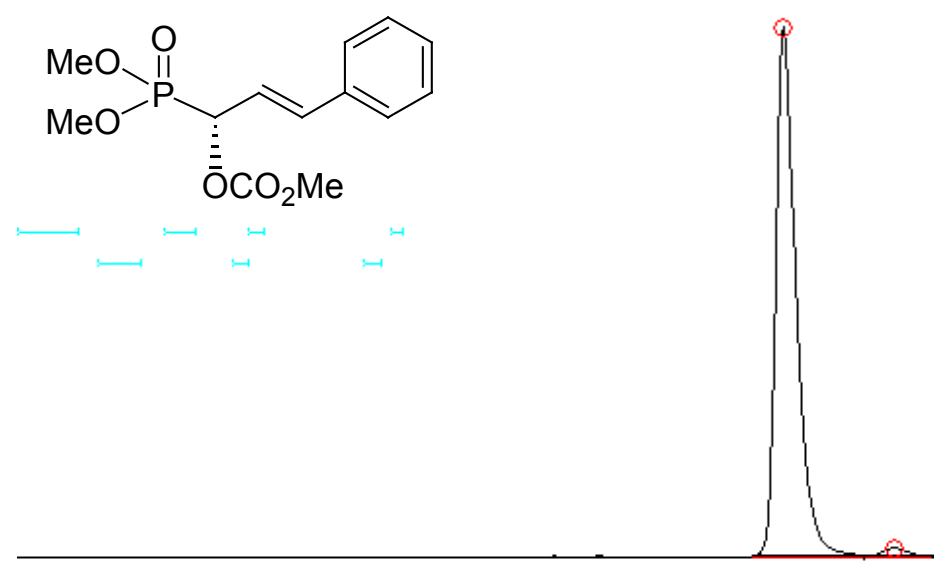

R Isomer (95\% e.e.)

\begin{tabular}{|c|c|c|c|}
\hline $\begin{array}{c}\text { Retention time } \\
(\mathrm{min})\end{array}$ & Area & \% Area & Ratio \\
\hline 12.35 & 27003 & 97.5 & 39 \\
\hline 14.15 & 681 & 2.5 & 1 \\
\hline
\end{tabular}


Column-ChiralPak AS, EtOH:hexane (20:80), $1 \mathrm{~mL} / \mathrm{min}$, det $210 \mathrm{~nm}$

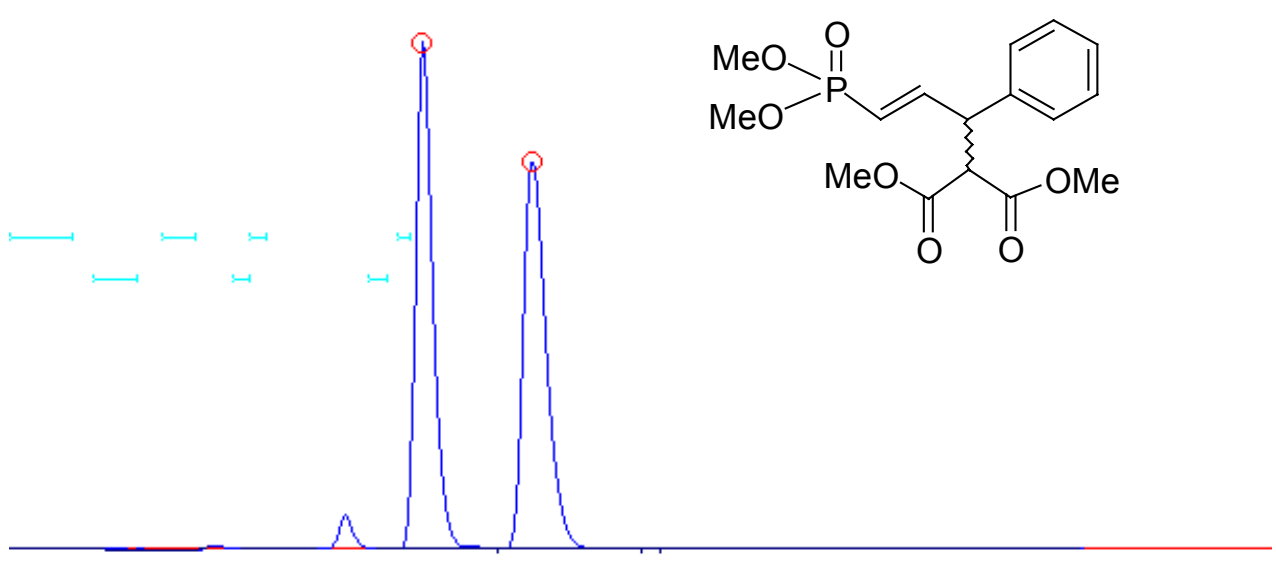

\section{Racemic}

\begin{tabular}{|c|c|c|}
\hline $\begin{array}{c}\text { Retention time } \\
\text { (min) }\end{array}$ & Area & \% Area \\
\hline 6.38 & 34130 & 49.9 \\
\hline 8.10 & 34349 & 50.1 \\
\hline
\end{tabular}

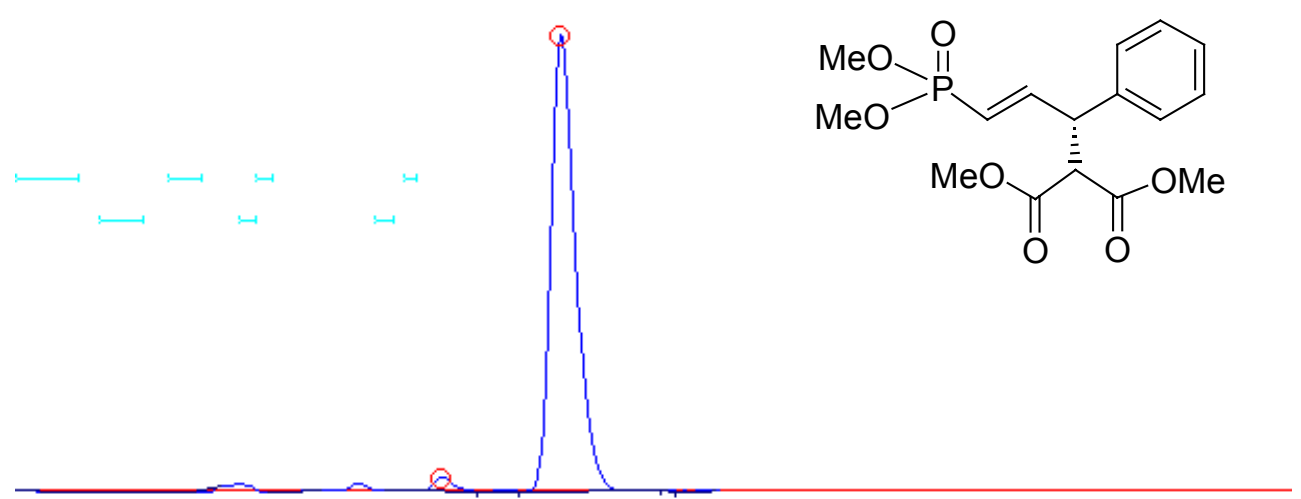

R Isomer (95\% e.e.)

\begin{tabular}{|c|c|c|c|}
\hline $\begin{array}{c}\text { Retention time } \\
\text { (min) }\end{array}$ & Area & \% Area & Ratio \\
\hline 6.58 & 333 & 2.3 & 42 \\
\hline 8.43 & 14210 & 97.7 & 1 \\
\hline
\end{tabular}


Column-ChiralPak AS, EtOH:hexane (20:80), $1 \mathrm{~mL} / \mathrm{min}$, det $210 \mathrm{~nm}$
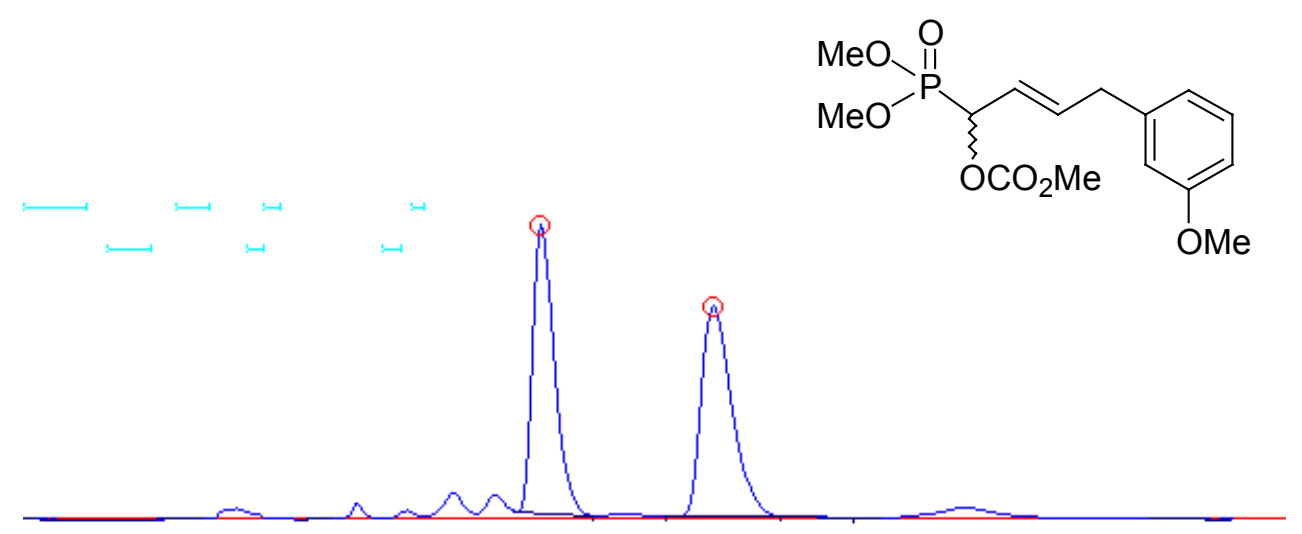

Racemic

\begin{tabular}{|c|c|c|}
\hline $\begin{array}{c}\text { Retention time } \\
(\mathrm{min})\end{array}$ & Area & \% Area \\
\hline 8.02 & 12114 & 49.14 \\
\hline 10.7 & 12536 & 50.86 \\
\hline
\end{tabular}

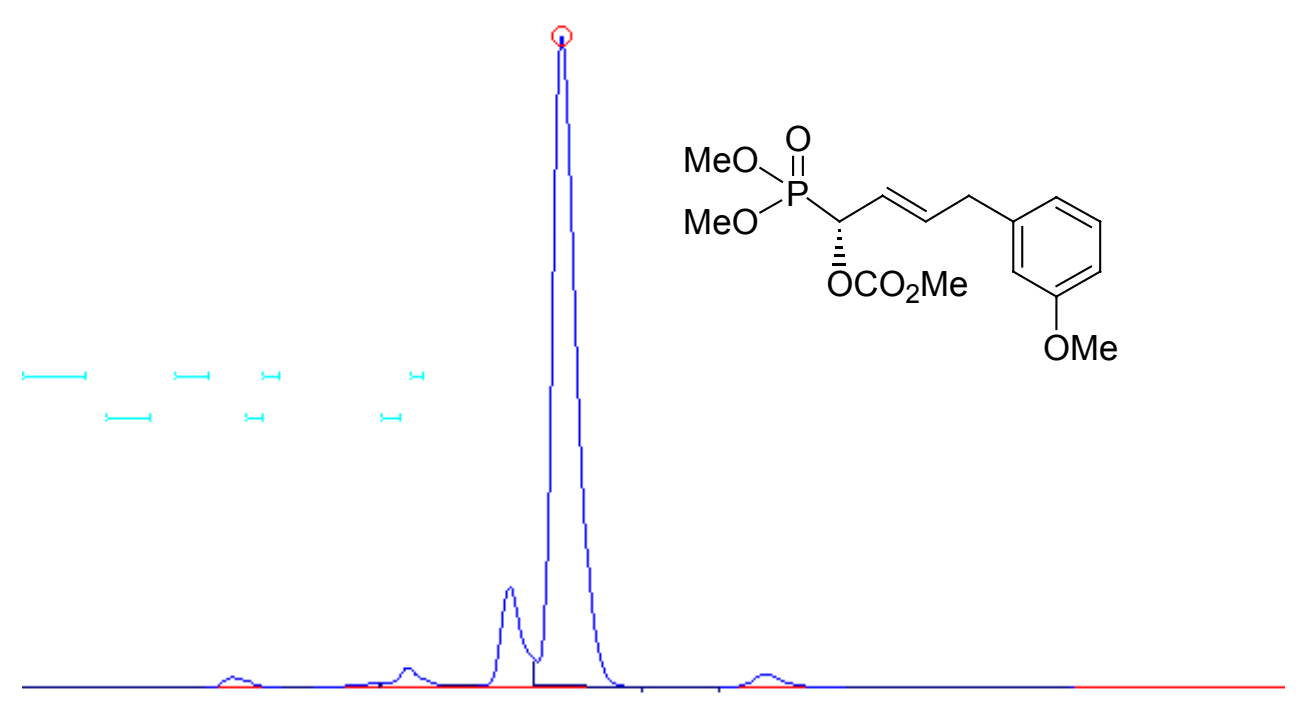

R Isomer (95\% e.e.)

\begin{tabular}{|c|c|c|c|}
\hline $\begin{array}{c}\text { Retention time } \\
\text { (min) }\end{array}$ & Area & $\%$ Area & Ratio \\
\hline 8.35 & 30870 & 97.69 & 41 \\
\hline 11.5 & 753 & 2.31 & 1 \\
\hline
\end{tabular}


Column-Chirobiotic-T, EtOH:hexane (50:50), $0.6 \mathrm{~mL} / \mathrm{min}$, det $210 \mathrm{~nm}$
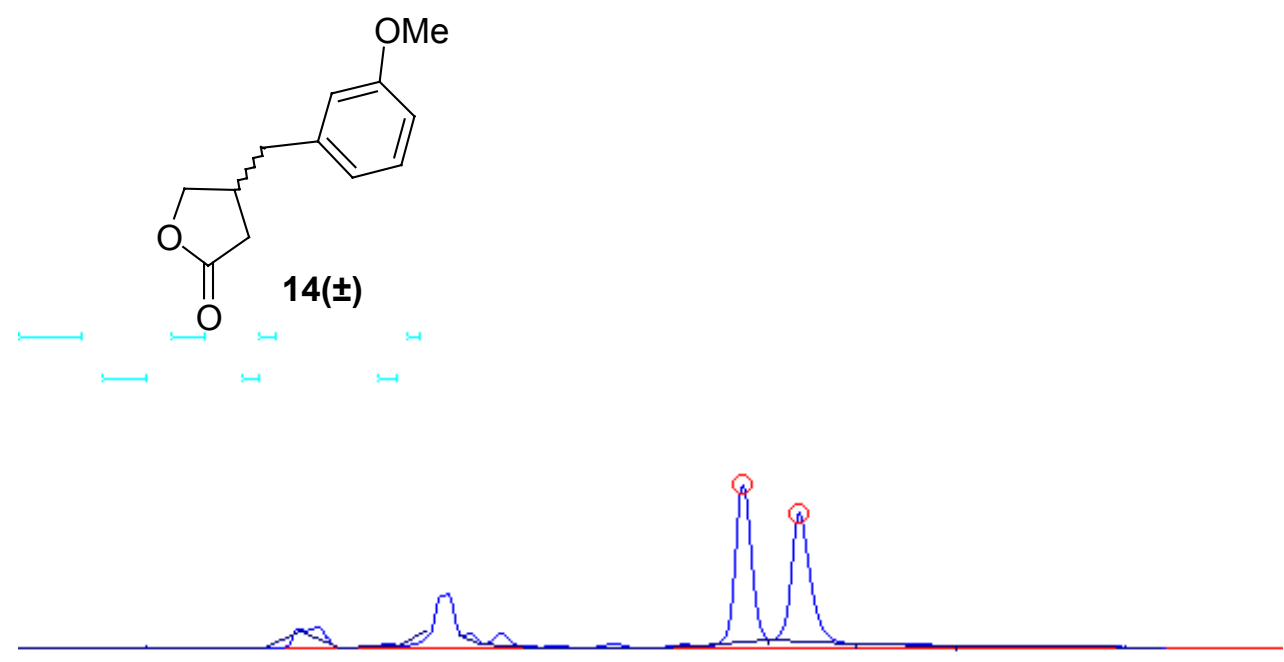

Racemic

\begin{tabular}{|c|c|c|}
\hline $\begin{array}{c}\text { Retention time } \\
(\mathrm{min})\end{array}$ & Area & \% Area \\
\hline 11.2 & 599 & 49.9 \\
\hline 12.1 & 603 & 50.1 \\
\hline
\end{tabular}
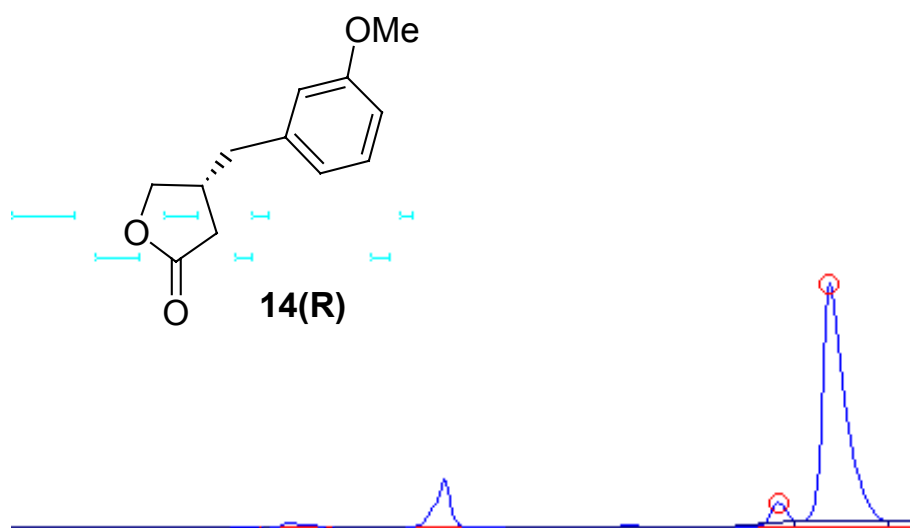

R Isomer $(92 \%$ e.e. $)$

\begin{tabular}{|c|c|c|c|}
\hline $\begin{array}{c}\text { Retention time } \\
(\mathrm{min})\end{array}$ & Area & $\%$ Area & Ratio \\
\hline 11.4 & 223 & 3.7 & 26 \\
\hline 12.2 & 5802 & 96.3 & 1 \\
\hline
\end{tabular}

